\title{
Sozialer Wandel der Familie im Strafrecht
}

\author{
Edward Schramm"
}

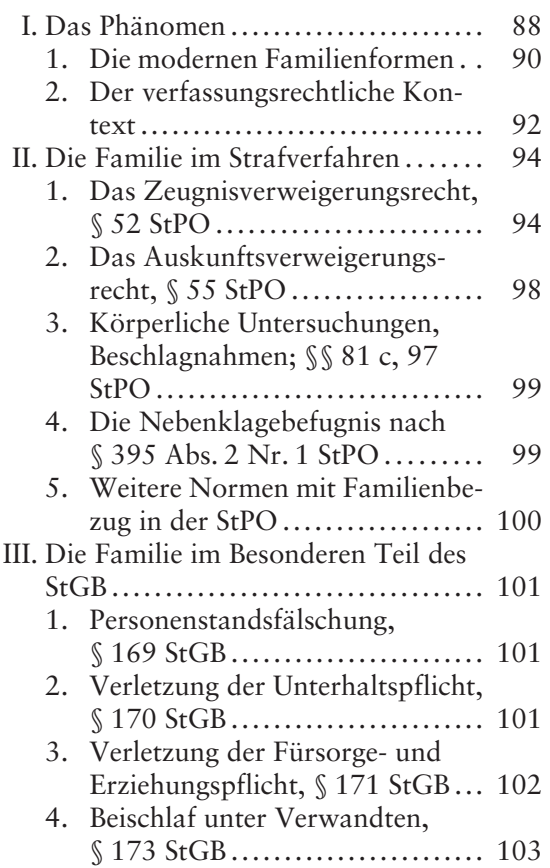

5. Sexueller Missbrauch von Schutzbefohlenen, $\$ 174$ StGB . . 104

6. Förderung der sexuellen Handlungen Minderjähriger, $\$ 180$ StGB ..................... 104

7. Aussetzung, $\mathbb{} 221$ Abs. 2 StGB . 106

IV. Die Familie im Allgemeinen Teil des

StGB .......................... 107

1. Der Angehörigenbegriff ......... 107

a) Systematik ............... 107

b) Analogiefähigkeit des Angehörigenbegriffs ............. 108

2. Gewaltverbot in der Familie; stellvertretende Einwilligung.... 110

a) Recht auf gewaltfreie Erziehung ..................... 110

b) Stellvertretende Einwilligung ..................... 111

3. Garantenstellungen, $\mathbb{\$} 13$ StGB . 111

a) Garantenstellung der Eltern gegenüber dem Kind........ 112

b) Verhältnis des Kindes gegenüber Eltern.................. 114

c) Verhältnis der Geschwister untereinander............... 115

V. Fazit und Ausblick................. 116

\section{Das Phänomen}

Der Begriff der Familie und die rechtlichen Erwartungen an das Zusammenleben in der Familie waren, wie die Rechtsgeschichte zeigt, niemals statisch. ${ }^{1}$ Doch seit den 1960 er Jahren des vergangenen Jahrhunderts unterliegen die Familienstrukturen in den meisten westlich geprägten Gesellschaften einem besonders tiefgreifenden Wandel. ${ }^{2}$ Bis dahin herrschte als normatives Leitbild die - in den Ehe- und Familienvorstellungen des späten 18. und 19. Jahrhunderts wurzelnde - sog. bürgerliche Familie vor, bestehend aus einem verheirateten Paar mit ihren leiblichen Kindern und mit

* Prof. Dr. Edward Schramm ist Universitäts-Professor an der Friedrich-Schiller-Universität Jena und Inhaber des Lehrstuhls für Strafrecht, Strafprozessrecht, Wirtschaftsstrafrecht, Europäisches und Internationales Strafrecht.

1 Vgl. Beck-Gernsheim, Was kommt nach der Familie? 2010; Rauchfleisch, Alternative Lebensformen, 1999; Schramm, Ehe und Familie im Strafrecht, S. 39 ff.; Schwab, Familie. in: Geschichtliche Grundbegriffe. Historisches Lexikon zur politisch-sozialen Sprache in Deutschland. Hrsg. von Otto Brunner, Werner Conze, Reinhart Kosselleck. Bd. 2 E - G. Stuttgart 1975, S. 266 ff.; BMFSFJ (Hrsg.), Siebter Familienbericht der Bundesregierung, 2006, BT-Dr. 16/1360. .

2 Vgl. nur Böhnisch/Lentz, Familien, 2. Aufl. 1999; Peukert, Familienformen im sozialen Wandel, 8. Aufl. 2012. 
strikter Rollenverteilung der Aufgaben (meist der Mann als Alleinverdiener, die Frau als Mutter zu Hause). Als literarischer „Beweis“ dafür soll den nachstehenden Überlegungen ein Auszug aus Schillers 1799 verfasstes „Lied von der Glocke“ vorangestellt werden ${ }^{3}$ - ein Gedicht, das lange zum Kanon der deutschen Literatur zählte, und das $A b$ - und vielfach auch Vorbild eines bis weit in das 20. Jahrhundert vorherrschenden Familienideals und Rollenverständnisses war, heute freilich aufgrund seines Inhalts in den Augen vieler (wenngleich bei weitem nicht aller) als „hoffnungslos veraltet" anzusehen wäre: ${ }^{4}$

(...) Der Mann muß hinaus

In's feindliche Leben,

$M u ß$ wirken und streben

Und pflanzen und schaffen,

Erlisten, erraffen,

$M u ß$ wetten und wagen

Das Glück zu erjagen.

$\mathrm{Da}$ strömet herbey die unendliche Gabe,

Es füllt sich der Speicher mit köstlicher Haabe,

Die Bäume wachsen, es dehnt sich das Haus.

Und drinnen waltet

Die züchtige Hausfrau,

Die Mutter der Kinder,

Und herrschet weise

Im häuslichen Kreise,

Und lehret die Mädchen

Und wehret den Knaben,

Und reget ohn' Ende

Die fleißigen Hände,

Und mehrt den Gewinn

Mit ordnendem Sinn,

Und füllet mit Schätzen die duftenden Laden,

Und dreht um die schnurrende Spindel den Faden,

Und sammelt im reinlich geglätteten Schrein

Die schimmernde Wolle, den schneeigten Lein,

Und füget zum Guten den Glanz und den Schimmer,

Und rubet nimmer.

Und der Vater mit frohem Blick,

Von des Hauses weitschauendem Giebel

Ueberzählet sein blühend Glück (... $)^{5}$

3 Zitiert nach der Erstausgabe: Friedrich Schiller, Das Lied von der Glocke, Musen-Almanach für das Jahr 1800, Tübingen 1800, S. $251-272$.

4 Vgl. nur Jens (Hrsg.), Kindlers Neues Literatur Lexikon, 1998, Bd. 14, S. 919.

5 Schiller hat die Fragilität dieses ehelichen und familiären Glücks (die Frau wird bald sterben) schon wenige Zeilen danach thematisiert: Doch mit des Geschickes Mächten / Ist kein ewger Bund zu flechten,/ Und das Unglück schreitet schnell. 
Ihren faktischen sozialen und zugleich normativen Vorrang haben eine so verstandene Ehe und Familie jedoch schon vor Jahrzehnten verloren. Veränderte soziale und ökonomische Rahmenbedingungen, die medizinische Entwicklung (vor allem die Entwicklung der Antibaby-Pille), die einsetzende und fortschreitende Emanzipation der Frauen, die erheblich erleichterte Form der Ehescheidung, die Verflechtung von Berufs- und Familienleben sowie die Emanzipationsbewegungen der gays, lesbians, bisexuals and transgenders haben die bürgerliche Ehe und Familie zwar nicht zurück-, geschweige denn verdrängt, die (weitgehende) Exklusivität ihres sozialen und (familien-)rechtlichen Status' aber aufgehoben. Es sind an ihre Seite neue Formen der Partnerschaft und Familie getreten, die faktisch, in der Gesellschaft, und rechtlich, vor allem auf der Verfassungsebene und namentlich im bürgerlichen Ehe- und $\mathrm{Fa}$ milienrecht, aber auch im Straf- und Strafverfahrensrecht neue Fragestellungen hervorgebracht, Herausforderungen begründet und teilweise auch zu Reaktionen des Gesetzgebers geführt haben.

Welche Schwierigkeiten diese Veränderungen bei der rechtlichen Bewertung auslösen, hat etwa Ludwig Salgo im Zusammenhang der sorgerechtlichen Fragestellungen ausgeführt: Es „(...) bestimmt das Zusammenleben in neu zusammengesetzten Familienformen eine hohe Komplexität und Vielfalt ("meine, deine, unsere Kinder"), so dass die Regulierungs- und Steuerungsfähigkeit des Rechts in diesem "Dschungel" von Beziehungen ("die Patchworkfamilie") schnell an Grenzen stoßen kann. Ein rechtlicher Rahmen, der flexibel genug ist, um den sehr unterschiedlichen tatsächlichen Lebenssituationen gerecht zu werden, scheint noch nirgends gefunden. " ${ }^{6}$ Mögen auch durch das Kindschaftsrechtsreformgesetz von 1998 und das Lebenspartnerschaftsgesetz von 2001 dem sozialen Wandel Rechnung tragende, insbesondere auch stieffamilienbezogene Regelungen in das BGB aufgenommen worden sein, so hat der Gesetzgeber gleichwohl bis heute keine umfassende familienrechtliche Regelung der Rechtsbeziehungen innerhalb der Stief- und Patchworkfamilie geschaffen und das Strafrecht nicht in sein Blickfeld genommen.

\section{Die modernen Familienformen}

Vor diesem Hintergrund sei in diesem Beitrag der Blick folgenden fünf „neuen“ Familienformen zugewandt, die als besonders bedeutsame Formen eines gewandelten Familienverständnisses angesehen werden: ${ }^{7}$

1. Die nichteheliche Lebensgemeinschaft, allerdings nur, soweit sie einen Familienbezug aufweist, $\mathrm{d}$. h. bei denen zumindest ein Partner zugleich Elternteil eines an der Lebensgemeinschaft partizipierenden Kindes ist; in Deutschland ist die Zahl

7 Vgl. etwa Peukert (o. Fn. 2), S. 77. 
der Familien, die aus einer nichtehelichen Lebensgemeinschaft bestehen, von 452.000 (1996) auf 743.000 (2011) gestiegen. ${ }^{8}$

2. Der alleinerziehende Elternteil, zuweilen als Ein-Eltern-Familie in Gestalt der Mutter- oder Vaterfamilie bezeichnet; hier ist die Zahl von 1.3 Millionen (1996) auf 1.6 Millionen (2011) angewachsen. ${ }^{9}$

3. Die Stieffamilie, auch häufig und inzwischen vorrangig Patchworkfamilie (oder zuweilen Folgefamilie) genannt. Darunter versteht man die Aufspaltung einer Familie 1. nach dem Tod eines Elternteils (als die „klassische Form“ der seit jeher bekannten Stieffamilie) oder nach der Trennung beider Elternteile (als die „moderne" Form der Stieffamilie. bei der beide biologischen Elternteile leben) und 2. im Anschluss daran die Anbindung der fragmentierten Familie an eine bereits bestehende Familie. Man rechnet damit, dass ca. 10 bis 14 Prozent aller Familien in Deutschland Patchworkfamilien sind, dass fast $11 \%$ der Kinder in solchen Familien aufwachsen und drei Viertel der Partner in Stief- und Patchworkfamilien miteinander verheiratet sind. ${ }^{10}$

4. Die Adoptivfamilie, bei der die soziale Elternschaft mit einer rechtlichen Anerkennung des angenommenen Kindes verbunden ist.

5. Die sog. Regenbogenfamilie, d. h. Familien, in denen Kinder mit zwei gleichgeschlechtlichen Elternteilen aufwachsen, von denen ein Elternteil im Regelfall die leibliche Mutter oder der leibliche Vater ist, sofern das Kind nicht adoptiert wurde (dann ist die Regenbogenfamilie zugleich eine Adoptivfamilie). Nach statistischen Erhebungen ist die Zahl der gleichgeschlechtlichen Lebensgemeinschaften mit Kind von 3000 (1996) auf 4000 (2011) angestiegen. ${ }^{11}$

Die eingetragene Lebenspartnerschaft, $d$. h. die förmlich registrierte Partnerschaft von lesbischen Frauen oder schwulen Männern, bedarf im Folgenden keiner vertieften Erörterung. Dieses lebenspartnerschaftliche (und in der Sache familienrechtliche) Statusverhältnis wurde im Jahr 2001 durch das Lebenspartnerschaftsgesetz $(\mathrm{LPartG})^{12}$ geschaffen. Durch das LPartG und die nachfolgenden Änderungsgesetze wurde die Lebenspartnerschaft zwar nicht auf dieselbe verfassungsrechtlichen Ebene wie die Ehe i. S. d. Art. 6 Abs. 1 GG gestellt, die weiterhin, auch vom BVerfG, als rechtlich geordnete Verbindung und Lebensgemeinschaft von Mann und Frau ver-

8 Familienreport 2012, hrsg. v. Ministerium für Familien Senioren, Frauen und Jugend, S. 14 auf der Grundlage von Statistiken (Mikrozensus) des Statistischen Bundesamtes.

9 Familienreport 2012, S. 14.

10 Familienreport 2012, S. 20. Dabei ist die Zahl der Patchworkfamilien und nichtehelichen Lebensgemeinschaften mit Kindern in den sog. „neuen“ Bundesländern erheblich größer als in den „alten“ Bundesländern; vgl. Stief- und Patchworkfamilien in Deutschland. Monitor Familienforschung. Hrsg. v. Bundesministerium für Familie, Senioren, Frauen und Jugend, Band 31, 2013.

11 Familienreport (Fn. 8).

12 LPartG vom 16.2.2001 (BGBl. I S. 266), zuletzt geändert durch Gesetz vom 7.5.2013 (BGBl. I S. 1122). 
standen wird. ${ }^{13}$ Jedoch auf der einfachrechtlichen Ebene ist sie der Ehe - wenngleich nicht zu $100 \%$, aber doch sehr weitgehend ${ }^{14}$ - nahezu gleichgestellt. Sie ist im Strafgesetzbuch und der Strafprozessordnung in den Angehörigenbegriff einbezogen $(\mathbb{1 1} \mathrm{StGB})$ und steht damit, auch in ihrer Vorstufe des Partnerschaftsversprechens (als Pendant zum Verlöbnis), auf Augenhöhe mit der Ehe. Eine Ausnahme bildet insoweit nur der Straftatbestand der Bigamie, $\mathbb{S} 171$ StGB, der das zivilrechtliche Doppeleheverbot strafrechtlich sanktioniert, während das zivilrechtliche Verbot der doppelten Lebenspartnerschaft oder der Lebenspartnerschaft neben der Ehe nicht mit einem gesonderten strafrechtlichen Verbot sanktioniert wird. ${ }^{15}$ Auch wenn es „prophetischer Gaben“ bedürfte, um die künftige politische und gesetzgeberische Entwicklung in diesem Bereich vorhersagen zu können, spricht doch vieles für die Annahme, dass es wohl nur eine Frage der Zeit ist, bis eine formalisierte gleichgeschlechtliche Beziehung rechtlich - und nicht nur im Volksmund - als „Homo-Ehe“ angesehen und der Ehe vollkommen rechtlich gleichgestellt bzw. womöglich sogar direkt unter den Ehebegriff (etwa des Art. 6 Abs. 1 GG) subsumiert wird. Die eingetragene gleichgeschlechtliche Partnerschaft ginge dann letztlich in der Ehe auf, als eine der Ehe-Varianten in der langen, dynamischen und wechselvollen Geschichte des Eherechts.

Versteht man unter Familie in ihrem Begriffskern „die Zusammengehörigkeit von zwei oder mehreren aufeinander bezogenen Generationen, die zueinander in einer Eltern-Kind-Beziehung stehen “, ${ }^{16}$ wird deutlich, dass bei einer kinderlosen Lebenspartnerschaft der Begriff Familie ebenso unangebracht ist wie bei einer kinderlosen Ehe oder Lebensgemeinschaft. Die Partner dieser Beziehungen sind dann zwar teilweise Angehörige im Kontext der jeweiligen einfachrechtlichen Angehörigenbegriffe und (im Falle der Ehe und Lebenspartnerschaft) zugleich Angehörige der Familie des Ehe- und Lebenspartners (vgl. etwa $\$ 11$ Abs. 1 LPartG); sie bilden aber, isoliert betrachtet innerhalb ihrer kinderlosen Beziehung als solcher, keine Gemeinschaft von Eltern und Kindern ${ }^{17}$ und folglich verfassungs- wie einfachrechtlich keine Familie.

\section{Der verfassungsrechtliche Kontext}

Die Einordnung von modernen Familienformen in den verfassungsrechtlichen Kontext stellt sich als eine komplexe Fragestellung dar. Im Zentrum steht dabei Art. 6 GG. Ehe und Familie stehen unter dem besonderen Schutze der staatlichen Ord-

$14 \mathrm{Zu}$ den wenigen Ausnahmefällen vgl. etwa das bislang eingetragenen Lebenspartner verwehrte gemeinsame Adoptionsrecht; dazu und zur Verfassungswidrigkeit des Verbots der Sukzessivadoption vgl. BVerfG NJW 2013, 847.

15 Schramm (Fn. 1), S. 386.

16 Böhnisch/Lenz (Fn. 2), S. 28.

17 Badura, in: Maunz/Dürig, GG, 69. Elfg. (2013), Art. 6 Rn. 60. 
nung, heißt es in dessen ersten Absatz. Zu klären ist: Was bedeuten Ehe und Familie, und worin besteht der besondere Schutz?

Für den Ehebegriff knüpft die Rechtsprechung des BVerfG und die h. M. an die familienrechtliche Ausgestaltung der Ehe in den $\mathbb{S} \$ 1310 \mathrm{ff}$. BGB an. Danach ist Ehe die rechtlich geordnete Form einer auf Dauer angelegten Verbindung von Mann und Frau, deren Eingehung auf der Willensübereinstimmung der Ehegatten beruht und des Ordnungselements der staatlichen Mitwirkung durch den Standesbeamten bedarf. ${ }^{18}$ Damit fallen die nichtehelichen Lebensgemeinschaften und die Lebenspartnerschaften aus dem Schutzbereich des Art. 6 Abs. 1 GG. Verfassungsrechtlich sind sie auf den „bloßen“ Schutz aus Art. 2 Abs. 1 GG und womöglich aus Art. 3 Abs. 3 GG angewiesen. ${ }^{19}$

Erheblich offener für moderne Lebensformen (und insoweit soziologisch geprägt) ist der Familienbegriff in Art. 6 Abs. 1 GG. Nach dem sog. sozialen Familienbegriff des BVerfG ist Familie die Gemeinschaft von Eltern und Kindern, wobei es nicht darauf ankommt, ob die Kinder von den Eltern abstammen und ob sie ehelich oder nichtehelich geboren wurden. ${ }^{20}$ Damit fallen in den Schutzbereich der Familie auch die „besondere Fürsorge- und Verantwortungsbeziehung zu (minderjährigen) Adoptiv-, ${ }^{21}$ Stief- und Pflegekindern “, mithin auch die nichteheliche Lebensgemeinschaft mit Kind und die Regenbogenfamilie. ${ }^{22}$ Dies steht übrigens im Einklang mit dem Familienschutz in Art. 8 EMRK, dessen weiter Familienbegriff sich ebenfalls auf de facto-Familien und sowie Familien gleichgeschlechtlicher Paare erstreckt. ${ }^{23}$ Dabei ist freilich eine Ungleichbehandlung von klassischen und modernen Familienformen möglich, sofern gewichtige Gründe für eine solche Differenzierung vorliegen. ${ }^{24}$ Biologistischer als der Familienbegriff ist allerdings der Begriff des Erziehungsrechts, wie er in Art. 6 Abs. 2 GG zum Ausdruck kommt. Danach gehören zu den Eltern die leiblichen Eltern sowie die Adoptierenden, nicht jedoch Pflegeeltern, der Vormund oder das allein sozial-familiäre Elternverhältnis. ${ }^{25}$

18 So die Definition bei Badura, in: Maunz/Dürig, 69. Elfg. 2013, Art. 6 GG Rn. 42.

19 Badura, in: Maunz/Dürig (Fn. 11) Art. 6 GG Rn. 55.

20 BVerfGE 108, 82 Rn. 89; Wellenhofer, Familienrecht, 2. Auflage 2011, $\$ 2$ Rn. 3; Schwab, Familienrecht, 21. Aufl. 2013, $\$ 2$ Rn. 16.

21 BVerfGE 80, 81 Rn. 33.

22 So explizit BVerfG NJW 2013, 847 Rn. 64: „Angesichts des Schutzzwecks des Familiengrundrechts ist auch eine aus gleichgeschlechtlichen Lebenspartnern und einem Kind bestehende, dauerhaft angelegte, sozial-familiäre Gemeinschaft eine Familie im verfassungsrechtlichen Sinne“; ähnlich bereits BVerfG NJW 2011, 988 Rn. 27.

23 Vgl. Mayer-Ladewig, EMRK, 3. Aufl. 2011, Art. 8 Rn. 49.

24 BVerfGE 106, 166.

25 Vgl. Badura, in: Maunz/Dürig, Art. 6 GG Rn. 99. Aufgrund der offenen Formulierung der „Eltern“ auch das Elternrecht nach Art. 6 Abs. 2 GG auf Gemeinschaften von zwei gleichgeschlechtlichen Personen und leiblichen/adoptierten Kind(ern) erstreckt werden. 
Als Fazit steht damit fest, dass die modernen „sozialen“ Familienformen im Regelfall in den Schutzbereich des Art. 6 Abs. 1 GG fallen und insofern prinzipiell an denselben verfassungsrechtlichen Garantien partizipieren, die für die klassische Familie vorgesehen sind. Dies bedeutet dreierlei: Art. 6 Abs. 1 GG enthält die Erklärung, dass der Familie ein besonders positiver Wert zukommt, aus dem eine Schutzverpflichtung des Staates folgt. Der Staat hat sodann die Aufgabe, die Familie vor Übergriffen durch den Staat zu schützen, d. h. der Staat unterwirft sich verfassungsrechtlich einer Selbstbindung, gewisse familiäre Freiheitssphären zu respektieren und die Regelungen im Innenverhältnis den Familienangehörigen zu überlassen. Sodann obliegt es den Staat, die Familie vor Übergriffen von innen (durch andere Familienmitglieder) oder von außen (durch Familienexterne) zu schützen. ${ }^{26}$

Damit ergeben sich in einer verfassungsrechtlichen orientierten Systematik familienbezogener Strafvorschriften folgende zwei Kategorien von Normen: Es kann zwischen solchen Vorschriften differenziert werden, mit denen das Strafrecht positiv Rechtsgüter aus dem Bereich der Familie schützt - Schutz durch Strafe -, und solchen, die auf eine negativ-abwehrende Wirkung gegen den Staat, d. h. auf den Schutz vor Schädigungen und Gefährdungen von Familien durch die staatliche Strafgewalt ausgerichtet sind - Schutz vor Strafe.$-{ }^{27}$ Dieser Schutz vor dem und durch das Strafrecht kann gleichermaßen von der klassischen Kernfamilie wie von der Patchwork-Familie oder Regenbogenfamilie für sich in Anspruch genommen werden.

\section{Die Familie im Strafverfahren}

Familienangehörige können Verdächtige oder Verletzte einer Straftat sein, die Rolle des Zeugen in einem Strafprozess spielen oder eine förmliche Funktion im Strafverfahren innehaben, etwa als Richter oder Staatsanwälte, eine Straftat zum Nachteil ihrer Angehörigen begangen haben oder als Nebenkläger in Betracht kommen. Hier bedarf es der Klärung, inwieweit die Angehörigen einer der genannten neuen Familienformen einen Status innehaben, der denjenigen einer klassischen Familie entspricht.

\section{Das Zeugnisverweigerungsrecht, $\S 52$ StPO}

Das familiäre Beziehungsgefüge ist besonders starken Belastungen ausgesetzt, wenn sich ein Strafverfahren gegen einen Beschuldigten richtet, hinsichtlich dessen Tat ein Angehöriger als Zeuge in Betracht kommt. In dem hier interessierenden Zusammenhang sind Fälle von Interesse, in denen beispielsweise ein Stiefvater seinen minderjährigen Sohn dabei beobachtet, wie er Personen im Internet beleidigt, Urheberrechtsverletzungen begeht oder mit Betäubungsmitteln handelt. Aber auch der um-

26 Schramm (Fn. 1), S. 24.

27 Schramm (Fn. 1) S. 27 und ders., JA 2013, 881, in Anlehnung an Schünemann, Festschrift für Pawlowski, 1996, S. 275, 283. 
gekehrte Fall ist sehr gut denkbar, dass etwa das Stiefkind solche Verhaltensweisen bei seinem Stiefvater feststellt und seine Beobachtungen dann folgerichtig und wahrheitsgemäß - mit entsprechender strafrechtlicher Sanktionierung unter den Voraussetzungen des $\mathbb{S} 153,154 \mathrm{StGB}$ - als Zeuge gegenüber den Strafverfolgungsorganen zu offenbaren hätte.

Nach $\ 52$ Abs. 1 StPO haben neben den Verlobten, Ehegatten und Lebenspartner unter anderem diejenigen Familienangehörigen ein Zeugnisverweigerungsrecht, die mit dem Beschuldigten in gerader Linie verwandt sind, d. h. die i. S. d. $\$ 1589$ BGB voneinander abstammen. ${ }^{28}$ Darunter fallen die Kinder in einer nichtehelichen Lebensgemeinschaft, soweit die Partner auch die leiblichen Eltern des Kindes sind; ebenso adoptierte Kinder sind i. V. m. $\$ 1754$ BGB einbezogen. Stiefkinder fallen dagegen - formal betrachtet - direkt erst dann in den Schutzbereich des $\$ 52 \mathrm{StPO}$, wenn der leibliche Vater bzw. die leibliche Mutter die Stiefmutter bzw. den Stiefvater geheiratet hat: Dann sind Stiefkind und Stiefvater bzw. Stiefmutter gem. 1590 BGB miteinander verschwägert ${ }^{29}$ und besitzen demenentsprechend ein Zeugnisverweigerungsrecht. ${ }^{30}$ Dagegen sind diejenigen Kinder, die in einer Patchworkfamilie oder einer Regenbogenfamilie aufwachsen, ohne vom nichtleiblichen Elternteil adoptiert worden zu sein oder ohne dass eine Heirat stattgefunden hat, auf den ersten Blick nicht zeugnisverweigerungsberechtigt.

Die herrschende Meinung lehnt eine analoge Anwendung des $₫ 52$ Abs. 1 StPO auf die darin nicht genannten faktischen Angehörigenverhältnisse, meist nur explizit auf nichteheliche Lebensgemeinschaften - in der Sache damit aber auch auf Pflege-, Patchwork- oder Regenbogenkindschaftsverhältnisse - ab. Das Gesetz beschreibe bewusst abschließend den Kreis der Zeugnisverweigerungsberechtigten; es liege gerade keine Regelungslücke vor. ${ }^{31}$ Es fehle bei solchen Beziehungsformen der Wille zu einer auf die rechtsinstitutionell anerkannte und daher allein schutzwürdige eheliche Bindung; enge persönliche Beziehungen als solche (wie z. B. die nahe Freundschaft) würden auch sonst nicht geschützt. ${ }^{32}$

Gegen die vorherrschende Rechtsansicht ist jedoch einzuwenden, dass die momentane Rechtslage bzw. - praxis zumindest dann unbefriedigend ist, wenn es gute Gründe dafür gibt, dass der Stiefvater oder die Stiefmutter (bzw. der Regenbogenvater oder die Regenbogenmutter) das Kind nicht adoptieren möchten (etwa um die

28 Zum entsprechenden Zeugnisverweigerungsrecht des Gutachters vgl. \$76 Abs. 1 StPO; zur Nichtverlesbarkeit von Zeugenaussagen bei Zeugnisverweigerung erst im Hauptverfahren $\mathbb{2} 252$ StPO; zum Eidesverweigerungsrecht der Angehörigen $\$ 61$ StPO.

29 Vgl. BayObLG Beschl. vom 19.3.2001 - 5St RR 40/01.

30 LG Berlin FamRZ 2004, 905.

31 Vgl. Kindhäuser, Strafprozessrecht, 3. Aufl. 2013, \$21 Rn. 48;Kudlich/Roy, JA 2003, 565;MeyerGoßner/Schmitt, StPO, 56. Aufl. 2013, \$52 Rn. 6; Senge, KK-StPO, 7. Aufl. 2013, $\$ 52$ Rn. 11 (nur bei „gesetzlichen Angehörigenverhältnissen“).

32 Roxin/Schünemann, Strafverfahrensrecht, 27. Aufl. 2012, $\$ 26$ Rn. 15. 
Bindung des Kindes zum leiblichen Elternteil nicht zu [zer-]stören) oder wenn es ihnen rechtlich gar nicht möglich ist. Auch hat es das Kind einer Patchworkfamilie nicht in der Hand, ob die neue Beziehung des einen Elternteils mit einer EheschlieBung usw. rechtsinstitutionell abgesichert wird und damit formal ein Angehörigenverhältnis begründet wird. Dagegen können die beiden Partner eigenverantwortlich darüber befinden, ob sie ihre Beziehung mit einem Verlöbnis, einer Ehe oder Lebenspartnerschaft absichern und so ein Angehörigenverhältnis begründen. Auch stehen bei der Frage des Zeugnisverweigerungsrecht in nichtehelichen Lebensgemeinschaften andere Interessen auf dem Spiel als im Verhältnis des Patchworkelternteils zum Kind: Es geht hier nicht um den Fortbestand einer partnerschaftlichen Beziehung, sondern um das ungestörte Aufwachsen eines Kindes in einem neuen familiären Umfeld, um Empathie, individuelle Förderung, ein positives Familienklima, eine gelingende Sozialisation und eine unauffällige Entwicklung als zentrale Weichenstellungen und Fundament für das weitere Leben des Kindes. ${ }^{33}$ Die hier tangierten Individual- und Kollektivinteressen erfordern eine besondere Unterstützung.

Abgesehen davon kann der dem Gesetz zugrundeliegende Gewissenskonflikt, entweder wahrheitsgemäß aussagen oder die Unwahrheit sagen zu müssen, genauso gut für ein Stiefkind bestehen - und erst recht für einen Stiefelternteil, wenn er Zeuge einer von Stiefkind begangenen Straftat wird. Angesichts der, wie eingangs gezeigt, stetig zunehmenden Zahl von Patchworkfamilien ohne verheiratete Partner handelt es sich dabei nicht um eine praktisch zu vernachlässigende Problematik. Der österreichische Gesetzgeber hat darauf bereits reagiert und den für das Zeugnisverweigerungsrecht ( $\$ 156$ Abs. 1 Nr. 1 österreichische StPO) maßgeblichen Angehörigenbegriff auf die nichteheliche Lebensgemeinschaft und die Kinder und Enkel eines der nichtehelichen Partner erstreckt ( $\mathbb{7 2}$ Abs. 2 österreichisches StGB). ${ }^{34}$ Die deutsche Legislative wäre an sich daher „dringend aufgerufen, den Schutzbereich der Vorschrift heutigen gesellschaftlichen Verhältnissen anzupassen " (Kett-Straub), ${ }^{35}$ wobei hier, wie von Kett-Straub vorgeschlagen, durchaus im künftigen Gesetz eine Orientierung an dem inhaltlichen Kern des Ehebegriffs erfolgen könnte. Ein etwaiger Verweis auf den Aussagenotstand in $\$ 157$ StGB wäre übrigens keine überzeugende Lösung, da diese Strafzumessungsregel nicht nur an enge Voraussetzungen geknüpft ist, sondern darüber hinaus nur eine - zudem fakultative - Strafmilderung vorsieht.

33 Zu Familienbeziehungen in Stief- und Patchworkfamilien aus Sicht der Kinder vgl. Stief- und Patchworkfamilien in Deutschland (o. Fn. 10), S. 19.

34 \ 72 Abs. 2 ÖStGB lautet: „Personen, die miteinander in Lebensgemeinschaft leben, werden wie Angehörige behandelt, Kinder und Enkel einer von ihnen werden wie Angehörige auch der anderen behandelt."

35 Kett-Straub, ZRP 2005, 50; bei dauerhaften Lebensgemeinschaften Beulke, Strafprozessrecht, 12. Aufl. 2012, $\mathbb{1 0}$ Rn. 191; Skwirblies, Nichteheliche Lebensgemeinschaft und Angehörigenbegriff im Straf- und Strafprozeßrecht, 1990, S: 199 - 203. 
Richtigerweise sollten sich Pflege- Patchwork- und Regenbogenkinder und Pflege-, Patchwork- und Regenbogeneltern zu Gunsten des jeweils anderen dann auf ein Zeugnisverweigerungsrecht berufen dürfen, wenn zwischen ihnen eine Beziehung gelebt wird, die das gleiche Bedürfnis nach dem Schutz vor der staatlichen Strafgewalt auslöst wie das „natürliche“ Eltern- und Kindverhältnis, mithin ebenso auf Solidarität, Fürsorge, innere Bindung und Dauer angelegt ist.

Damit stellt sich die Frage, ob die Rechtsprechung sich mit dem gegenwärtigen Gesetzestand und der Passivität des Gesetzgebers abfinden muss - und darf. Die Antwort dürfte im Lichte der Verfassungsbindung der Rechtsprechung eher „Nein“ lauten. Solange der Gesetzgeber aber nicht aktiv wird, ist im Wege einer verfassungskonformen Interpretation des $\$ 52$ Abs. 1 StPO die Norm analog auf eine kernfamilienäquivalente, $d$. h. eine tatsächliche Familiengemeinschaft darstellende Stieffamilie anzuwenden, in dem das Verhältnis des Kindes zum Stiefelternteil demjenigen einer intakten Beziehung eines Kindes zu seinem leiblichen Elternteil entspricht. Auch diese Familienform genießt über Art. 6 Abs. 1 GG den besonderen Schutz der Verfassung; nichts anderes kann für die Regenbogenfamilie gelten.

Darüber hinaus streitet auch der Gleichheitssatz in Art. 3 Abs. 1 GG für eine Gleichbehandlung, der zugleich die materielle Legitimation, aber auch die Begrenzung für eine analoge Anwendung des $\mathbb{5} 52$ StPO gibt: Man wird, in Anlehnung an KettStraub, nur eine dem natürlichen Eltern-und Kind-Verhältnis äquivalente Patchwork-Familienstruktur genügen lassen können. ${ }^{36}$ Besteht zwischen dem in die Patchworkfamilie aufgenommenen Kind und dem neuen Partner bzw. dessen Kindern keine tatsächlich praktizierte familiäre Gemeinschaft, sondern etwa nur eine Art Wohn- oder reine Zweckgemeinschaft, oder lehnt der neue Partner das Kind ab (oder umgekehrt), muss eine analoge Anwendung des $\$ 52$ StPO ausgeschlossen sein. Darüber hinaus ist zu beachten, dass Stiefkinder durchaus anfällig sind für Straftaten, die zu ihrem Nachteil vom Stiefelternteil begangen werden; man denke nur an Sexualstraftaten oder Gewaltdelikte. ${ }^{37}$ In solchen Fällen wäre dann, wie auch im Falle der Schwägerschaft nach $\mathbb{1} 1590$ BGB, im Rahmen des $\mathbb{5} 52$ Abs. 2 S. 2 StPO ein Ergänzungspfleger zu bestellen. ${ }^{38}$

Man mag gegen den hier vertretenen Lösungsansatz einwenden, dass er zu Rechtsunsicherheit führe, da mit dem formalen Angehörigenstatus ein sicheres Kennzeichen für ein Zeugnisverweigerungsrecht gegeben sei, das bei einer materiellen Bestimmung des Angehörigenverhältnisses eben fehle. Ebenso sind Missbräuche des Zeugnisverweigerungsrechts durch Mitglieder der Patchworkfamilie denkbar. Wenn freilich der

36 Kett-Straub, ZRP 2003, S. 50.

37 Beispiele hierfür bei VG Berlin, Urteil vom 17.9.2012 - VG 80 K 10.12 OL (=BeckRS 2012, 60672); BGH, Beschl. vom 12.10.2000- 5 StR $397 / 00$ (= BeckRS 200030136381 ).

38 Vgl. LG Berlin FamRZ 2004, 905. 
Strafrichter nach den entsprechenden Einlassungen und Erklärungen zu der wohlbegründeten Überzeugung gelangt, dass die Zwangslage, die mit dem Zeugnisverweigerungsrecht erfasst werden soll, ebenso für das Patchworkkind oder den Patchworkelternteil bestehen könnte, spricht alles dafür, eine analoge Anwendung des $\$ 52$ StPO - ausnahmsweise - zu bejahen.

Dieser Betrachtungsweise steht auch nicht die Entscheidung des Bundesverfassungsgerichts aus dem Jahre 1999 entgegen, wonach bei einer engen freundschaftlichen Beziehung außerhalb einer noch bestehenden Ehe kein Zeugnisverweigerungsrecht bestünde. ${ }^{39}$ Zwar hat das BVerfG hier maßgeblich das formale Argument hervorgehoben, dass $\$ 52$ StPO nicht zur Anwendung gelangen solle, wenn der Beschuldigte noch verheiratet sei. Es hat aber keine Aussage darüber getroffen, wie es entschieden hätte, wenn die Beschuldigte bereits geschieden gewesen wäre, sie aber mit dem Zeugen noch nicht verheiratet oder verlobt gewesen wäre. Vielmehr hat das BVerfG durchblicken lassen, dass materielle Gesichtspunkte Bedeutung erlangen können, wenn das Gericht hervorhebt, dass die Ehe eine Lebensgemeinschaft zwischen einem Mann und einer Frau sei, die auf Dauer angelegt sei, daneben keine weitere Lebensgemeinschaft gleicher Art zulasse und über die Beziehungen in einer reinen Haushalts- und Wirtschaftsgemeinschaft hinausgehe. Man gewinnt hier eher den Eindruck, dass das BVerfG nur deshalb eine analoge Anwendung des $\mathbb{5} 52$ StPO abgelehnt hatte, weil die Beschuldigte noch verheiratet war.

\section{Das Auskunftsverweigerungsrecht, $§ 55 \mathrm{StPO}$}

Sofern kein Zeugnisverweigerungsrecht besteht, können trotzdem Situationen im Gerichtssaal auftreten, in denen in einer Zeugenaussage Dinge zur Sprache gebracht werden, die Angehörige einem Strafverfolgungsrisiko aussetzen würden. Um den Zeugen diese seelische Zwangslage zu ersparen und dessen inneren Zwiespalt zwischen gesetzlicher Zeugenpflicht und persönlichen Interessen aufzuheben, ${ }^{40}$ räumt $\$ 55 \mathrm{StPO}$ ihm auf der Grundlage der verfassungs- und völkerrechtlich garantierten Selbstbelastungsfreiheit ${ }^{41}$ das Recht ein, bei einer Aussage weder sich selbst noch einen Angehörigen i. S. d. $\$ 52 \mathrm{StPO}$, der nicht Beschuldigter ist, zu belasten. ${ }^{42} \mathrm{Nemo}$ tenetur se ipsum aut propinquum accusare. Damit tritt die gleiche Problematik wie bei $\mathbb{S} 52$ StPO auf. Man stelle sich den Fall vor, wie der nichtleibliche PatchworkVater eine Sachbeschädigung, etwa in Gestalt von Graffiti-Schmierereien, beobachtet und dabei erkennen muss, dass sein 16 jähriger Stiefsohn „Schmiere steht“. Darf dann der Patchwork-Vater bei der polizeilichen oder gerichtlichen Vernehmung verschweigen, dass er seinen Stiefsohn dort gesehen hat?

39 BVerfG NJW 1999, 1622; BVerfG, Beschl. v. 16.3.2001, 2 BvR 1598/00.

40 BGHSt 17, 245, 247.

41 BVerfG NJW 1975, 103.

42 Beulke, StPO, $\mathbb{} 10$ Rn. 195; Heger, StPO, 2013, Rn. 366; Kindhäuser, StPO, $\$ 21$ Rn. 52; MeyerGoßner, StPO, $\mathbb{} 55$ Rn. 1. 
Der Patchworkvater darf dann schweigen, wenn er die Mutter des jungen Mannes geheiratet hat, da er dann, wie bereits erwähnt, mit ihm gem. $\int 52$ Abs. 1 Nr. 3 StPO i. V. m. $\int 1590$ BGB verschwägert ist; das darf er hingegen nach herrschender Meinung mangels analoger Anwendbarkeit des $\mathbb{S} 55$ StPO nicht, wenn er dessen Mutter nicht geheiratet hat. Doch richtigerweise dürfte dann kein Grund bestehen, allein dem formalen Band der Ehe den Ausschlag zu geben, wenn das Verhältnis zwischen Patchworkvater und seinem Patchwork-Sohn dem „normalen“ Verhältnis zwischen einem Vater und seinem leiblichen Kind entspricht und daher die psychische Zwangslage für den Patchwork-Vater bei einer Zeugenaussage genau dieselbe wäre wie für den leiblichen Vater. Daher wäre es in solchen Fällen insgesamt konsequent, ein Aussageverweigerungsrecht des Stiefvaters (und im umgekehrten Falle des Stiefkinds) aus den gleichen Gründen anzunehmen, aus denen nach der hier vertretenen Ansicht auch ein entsprechendes Zeugnisverweigerungsrecht in dem oben skizzierten Sinne (analog) in Betracht kommt.

\section{Körperliche Untersuchungen, Beschlagnahmen; $§ \S 81$ c, 97 StPO}

Sodann können gem. $\int 81$ c Abs. 3 S. 1 StPO Zeugnisverweigerungsberechtigte Untersuchungen oder Entnahmen von Blutproben verweigern, d. h. wenn die Blutprobe im Strafverfahren zu Lasten des angehörigen Beschuldigten verwendet werden könnte. Auch hier ist über eine analoge Anwendung des $\mathbb{5} 52$ StPO ein Schutz von Stiefkindern oder Stiefeltern zu erreichen.

Nach $\ 97$ Abs. 1 StPO dürfen schriftliche Mitteilungen zwischen dem Beschuldigten und den nach $\ 52$ StPO zeugnisverweigerungsberechtigten Personen nicht beschlagnahmt werden. Die Ratio dieser Norm leuchtet unmittelbar ein: So wenig wie der Angehörige den Beschuldigten durch eine mündliche Aussage vor Gericht belasten muss, sowenig soll er durch schriftliche Beweisstücke zur Überführung seines Angehörigen beitragen. Da es auch hier eine untrennbare Verflechtung mit dem Kreis der in $\mathbb{5} 52$ StPO genannten Personen gibt, würde eine analoge Anwendung des $\mathbb{} 52$ StPO auf Verhältnis des Stief-/Patchworkvaters zum Stief/-Patchworkkind folgerichtig auch auf das Beschlagnahmeverbot des $\mathbb{} 97$ Abs. 1 StPO durchschlagen.

\section{Die Nebenklagebefugnis nach $§ 395$ Abs. 2 Nr. 1 StPO}

Im Falle eines Tötungsdelikts sieht $\$ 395$ Abs. 2 Nr. 1 StPO eine Nebenklagebefugnis der Kinder und der Eltern des Getöteten vor. Nach herrschender Meinung fallen darunter nur die leiblichen Kinder und die Eltern im biologischen und rechtlichen Sinne, nicht jedoch Stiefkinder und Stiefeltern, ${ }^{43}$ sofern der leibliche Elternteil den Stiefelternteil nicht geheiratet hat. Diese Gesetzeslage kann so nicht befriedigen, wenn man sich etwa einen auf fahrlässigem Verhalten eines Dritten beruhenden Unfall vorstellt, bei dem der alleinerziehende Patchworkvater den Tod seines geliebten 
Stiefkindes zu beklagen hat. Dass es hier ein elementares Bedürfnis nach Zulassung einer Nebenklage geben dürfte, liegt auf der Hand. Die Gesetzeslage ist insoweit genauso problematisch wie beim Zeugnisverweigerungsrecht. Hier ist unter dem Gesichtspunkt des Familienschutzes (Art. 6 Abs. 1 GG) und des Gleichbehandlungsgebots (Art. 3 Abs. 1 GG) eine analoge Anwendung der Norm auf Stiefväter, Stiefmütter oder Stiefkinder verfassungsrechtlich geboten, wenn die soziale und personale Verbundenheit zwischen dem Kind und dem Stiefelternteil derjenigen zwischen Kindern und ihren leiblichen Eltern entspricht, und die leiblichen Eltern oder einer dieser Elternteile zu dem leiblichen Kind in keiner nennenswerten persönlichen oder sozialen Verbindung mehr stehen. ${ }^{44}$

\section{Weitere Normen mit Familienbezug in der StPO}

Die Strafprozessordnung verwendet häufiger den Begriff des Angehörigen, ohne ihn durch den Zusatz „Angehöriger i. S. d. $\$ 52$ StPO“ oder, in Anlehnung an das materielle Strafrecht, mit dem Verweis auf den Angehörigen i. S. d. $\mathbb{} 11$ Abs. 1 StGB einzuschränken. Als Beispiele seien genannt $\mathbb{8} 87$ Abs. 4 StPO (Hinzuziehung eines Angehörigen bei Ausgrabung einer Leiche), $\mathbb{\$} 98$ Abs. 2 StPO (erforderliche gerichtliche Bestätigung einer Beschlagnahme, wenn kein Angehöriger zugegen war oder dieser widersprochen hat), $\mathbb{} 106$ StPO (wenn möglich Hinzuziehung eines erwachsenen Angehörigen bei Hausdurchsuchung), $\mathbb{S} \mathbb{S} 114$ b Nr. 6, 114 c StPO (Recht des Untersuchungshäftlings auf Benachrichtigung von Angehörigen), $\$ 286$ StPO (Angehörige als Vertreter des abwesenden Angeklagten), $\mathbb{} 397$ a Abs. 1 Nr. 2 StPO (Recht des Nebenklägers auf anwaltlichen Beistand, wenn Angehöriger eines Getöteten nach $\$ 395 \mathrm{StPO}$ ) oder $\mathbb{} \$ 56$ c Abs. 1 StPO (Aufschiebung eines Berufsverbots bei Härte für Angehörige).

Zwar ist bei diesen Regelungen durchaus im Einzelfall umstritten, ob nicht zuweilen doch auf den engen Angehörigenbegriff in $\$ 52$ StPO abgestellt werden muss. ${ }^{45} \mathrm{Zu}$ mindest erscheint es bei diesen Bestimmungen bereits nach dem Wortlaut der Norm nicht ausgeschlossen, Partner einer nichtehelichen Lebensgemeinschaft, aber auch Stiefkinder und Stiefeltern de lege lata in den Kreis der Angehörigen prinzipiell zu integrieren. Teilweise entschärft sich aber auch die Problematik, sofern neben den Angehörigen auch auf eine Vertrauensperson abgestellt wird (wie z. B. in $\mathbb{\$} 114$ b, 114 c StPO), da darunter, bei entsprechendem Vertrauensverhältnis, ${ }^{46}$ zwanglos auch Patchwork-Eltern und -kinder subsumiert werden können.

44 Weiner, in: Beck-OK-StPO, 17. Ed., $\$ 395$ Rn. 14.

45 So etwa bei der Exhumierung einer Leiche nach $₫ 87$ Abs. 4 S. 2 StPO Senge, KK-StPO, $\mathbb{\int} 87$ Rn. 8.

$46 \mathrm{Zu}$ den Anforderungen an die Vertrauensperson bei $\mathbb{} 114 \mathrm{c}$ StPO vgl. etwa Graf, KK-StPO, $\mathbb{S} 114 \mathrm{c}$ Rn. 6. 


\section{Die Familie im Besonderen Teil des StGB}

Im Besonderen Teil des StGB finden wir zahlreiche Straftatbestände, die insoweit einen Familienbezug besitzen, als in ihnen die Familie unmittelbar oder mittelbar geschützt wird. Im nachfolgenden Abschnitt sollen vor allem die vier familienbezogenen Straftatbestände aus dem 13. Abschnitt des Besonderen Teils des StGB mit der Überschrift „Straftaten gegen den Personenstand, die Ehe und Familie“ zur Diskussion stehen, nämlich die Personenstandsfälschung ( $\mathbb{S} 169$ StGB), die Verletzung der Unterhaltspflicht ( $\mathbb{S} 170$ StGB), die Verletzung der Fürsorge- und Erziehungspflicht

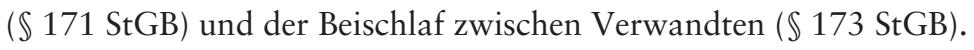

\section{Personenstandsfälschung, $§ 169$ StGB}

Den Kern der Norm bildet die im Einklang mit den familienrechtlichen Vorgaben des BGB stehende Registrierung bzw. Feststellung des familienrechtlichen Status' eines Menschen. ${ }^{47}$ Eine strafbare Personenstandsfälschung ist in Bezug auf Stieffamilien und nichtehelichen Lebensgemeinschaften mit Kindern ist daher nur denkbar, soweit diese Beziehungsformen oder einzelne Aspekte überhaupt in den Schutzbereich des außerstrafrechtlichen Personenstandsrechts fallen. Die Stieffamilie als solche wird aber ebenso wenig personenstandsrechtlich registriert wie die nichteheliche Lebensgemeinschaft, die Regenbogenfamilie oder die aufgrund Adoption ( $\mathbb{S} 7$ Abs. 6, 7 PStG) entstehende Familie aufgrund Lebenspartnerschaft, obgleich sie Familie im verfassungsrechtlichen Sinne sind. ${ }^{48}$ Nach $\mathbb{} 1$ PStG gehören zum Personenstand die Geburt, Eheschließung, Begründung einer Lebenspartnerschaft und der Tod sowie damit in Verbindung stehende familien- und namensrechtliche Tatsachen, nicht jedoch andere Daten. ${ }^{49}$ Folgerichtig dürfen in das Geburtenbuch $(\mathbb{S} 21$ Abs. 1 Nr. 4 PStG) nur die „Eltern“, und das sind die leiblichen Eltern, als solche eingetragen werden, da die rechtliche Abstammung die biologische, natürliche Abstammung nicht ersetzt. ${ }^{50}$ Eine Personenstandsfälschung kann aber im Zusammenhang mit einer modernen Familienform vorliegen, wenn dem Stiefvater ein Kind untergeschoben wird ( $\mathbb{S} 169$ Abs. 1 Var. 1 StGB) oder (i. S. d. $\mathbb{\int} 169$ Abs. 1 Var. 2 StGB) der Stiefvater das Stiefkind als eigenes ausgibt oder eine Regenbogenfamilie durch Falschangaben zur Familie i. S. d. $\$ 21$ PStG erklärt wird.

\section{Verletzung der Unterhaltspflicht, $§ 170$ StGB}

In $\mathbb{1} 170 \mathrm{StGB}$ wird die Nichterfüllung einer gesetzlichen Unterhaltspflicht mit einer Freiheitsstrafe bis zu drei Jahren bestraft, sofern diese mit einer Gefährdung des Lebensbedarfs des Unterhaltsberechtigten verbunden ist oder ohne die Hilfe Dritte ver-

47 Frommel, NK-StGB, $\mathbb{S} 169$ Rn. 2; Kindhäuser, StGB, $\mathbb{} 169$ Rn. 1; Schramm (Fn. 1) S. 314.

48 BVerfG NJW 2013, 847 Rn. 61.

49 Rhein, PStG, 2012, $\mathbb{S} 1$ Rn. 2.

50 LG Hamburg, Beschl. v. 4. 11. 2009, 301 T 596/09; die Verfassungskonformität bestätigend BVerfG NJW 2011, 988. 
bunden wäre. Im Rahmen des $\mathbb{1} 170$ StGB sind diejenigen Partner und Kinder praktisch „schutzlos“, denen kein Unterhaltsanspruch zusteht, wie etwa dem nicht adoptierten Stiefkind. Dem Strafrecht kann man diese „Strafbarkeitslücke“ - wenn man sie denn als eine solche einstufen möchte - nicht „vorwerfen“. Denn dies ist letztlich nur die Folge der gesetzgeberischen Entscheidung, $\mathbb{\$} 170$ StGB akzessorisch zum familienrechtlichen Unterhaltsrecht ausgestaltet zu haben. Die Strafbarkeit nach dieser Strafnorm setzt das Bestehen einer gesetzlichen Unterhaltspflicht voraus. Stiefeltern werden ihren Stiefkindern zwar vielfach de facto Unterhalt gewähren und sich moralisch als Unterhaltsschuldner empfinden; de jure aber sind sie zum Unterhalt in Deutschland nach dem BGB, anders als etwa in England oder den Niederlande, ${ }^{51}$ gerade nicht verpflichtet. ${ }^{52}$ Zwar kann sich der Stiefvater vertraglich zur Unterhaltszahlung verpflichten; aber das genügt angesichts des eindeutigen Wortlauts des Tatbestands gerade nicht. ${ }^{53}$ Strafrechtlich sanktioniert sind freilich gesetzliche Unterhaltspflichten wie der Unterhaltsanspruch des adoptierten Kindes ( $\$ 1751$ BGB).

\section{Verletzung der Fürsorge- und Erziehungspflicht, §171 StGB}

Die ungestörte Entwicklung eines Kindes in der Familie bis zum 16. Lebensjahr wird strafrechtlich gesondert über die Strafnorm des $\$ 171$ StGB geschützt. Die gröbliche Verletzung von Fürsorge- und Erziehungspflichten wird in $\mathbb{1 7 1}$ StGB mit einer Freiheitsstrafe bis zu 3 Jahren oder Geldstrafe pönalisiert, sofern die zu erziehende Person unter 16 Jahre alt ist und durch die Pflichtverletzung das Risiko bestimmter Entwicklungsstörungen - das Gesetz nennt etwa einen kriminellen Lebenswandel oder das Abgleiten in die Prostitution - geschaffen wird. Die Fürsorgekomponente meint in erster Linie den körperlichen aber auch seelischen Schutz des Kindes und die Erziehungskomponente die richtige Anleitung des Schutzbefohlenen in seiner körperlich-seelischen Entwicklung. ${ }^{54}$

Die für die Täterschaft vorausgesetzte Fürsorge- oder Erziehungspflicht kann auf Gesetz, Vertrag, öffentlich-rechtlichem Dienstverhältnis oder faktischer Übernahme beruhen. ${ }^{55}$ Fürsorgerisch tätig werden solche Personen, die dazu verpflichtet sind, ein Kind einerseits vor Schäden an Leib und Seele zu bewahren (und ihm deshalb z. B. keinen Alkohol verabreichen dürfen $)^{56}$ und andererseits dessen gesunde körperliche und seelische Entwicklung zu gewährleisten (Ernährung und „Wartung “ im weitesten Sinne, Versorgung im Krankheitsfall). Zu diesem Personenkreis gehören die Eltern aufgrund ihrer Pflicht zur Sorge um das Wohl ihres Kindes, wie sich aus Art. 6 Abs. 2 S. 1 GG (Pflicht zur Pflege) und $\$ 1626$ Abs. 1 S. 1, S. 2 Var. 1 BGB

51 Vgl. Mankowski, in: Staudinger, Art. 1 HÜU Rn. 104.

52 Engler, in: Staudinger, $\mathbb{1} 1601$ BGB Rn. 28; Monecke, MK-BGB, 6. Aufl. 2013, $\$ 1360$ a Rn. 11.

53 Fischer, StGB, 61. Aufl. 2014, $\$ 170$ Rn. 4; Schramm (Fn. 1) S. 348.

54 Neuheuser, NStZ 2000, 174.

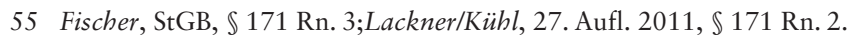

56 BGHSt 2, 348. 
(Pflicht zur Personensorge) ergibt. Eine Fürsorgepflicht haben auch der Vormund ( $\int 1793$ Abs. 1 S. 1 BGB), der Pfleger ( $\mathbb{1 9 1 5}$ Abs. 1 BGB) oder Pflegeeltern kraft Vertrags. ${ }^{57}$ Auch im öffentlich-rechtlichen Aufgabenbereich (z. B. Jugendarbeiter) oder sonstigen privaten Lebensbereich (z. B. nichteheliche Lebensgemeinschaft, Patchwork-Familie) können sich solche Pflichten ergeben. ${ }^{58}$ Wenn also die Patchwork- oder Regenbogenfamilie so ausgestaltet ist, dass der jeweilige Partner die Fürsorge und Erziehung für das Stiefkind mit übernommen hat, kann er sich auch nach \ 171 StGB strafbar machen. Mithin muß auch der Stiefvater, die Stiefmutter, die Partnerin der Mutter oder der Partner des Vaters als Fürsorge- und/oder Erziehungsverpflichteter dafür Sorge tragen, dass das Kind nicht der Gefahr einer erheblichen körperlichen oder seelischen Entwicklungsstörung, eines kriminellen Lebenswandels oder eines Abgleitens in die Prostitution ausgesetzt wird.

Die Gefahr einer seelischen Entwicklungsstörung besteht etwa dann, wenn in der Erziehung dem Tötungsverbot keine Bedeutung beigemessen wird; aber auch geschlechtlicher Verkehr eines Elternteils mit einem anderen Partner im Beisein des Kindes, ${ }^{59}$ das Anhalten zum Betteln ${ }^{60}$ oder der unkontrollierte Zugang zu jugendgefährdenden Gewalt- oder Pornographiedarstellungen fallen darunter. ${ }^{61}$ Hinsichtlich des Risikos der Prostitutionsausübung ist folgendes zu berücksichtigen: Zwar sind Verträge, die eine Prostitutionsausübung erwachsener Menschen zum Gegenstand haben, seit 2002 nach dem Prostitutionsgesetz ${ }^{62}$ ausdrücklich wirksam und damit die Ausübung der Prostitution als Beruf anerkannt. Wenngleich nicht mehr sittenwidrig, ist doch die Prostitutionsausübung weiterhin mit zahlreichen, ganz erheblichen Gefahren für Körper und Seele verbunden. Hinzu kommen z. B. das höhere Risiko sexueller Übergriffe, ein kriminogenes Umfeld, die Anfälligkeit der Prostitutionsausübung für Eigentums-, Vermögens- und Gewaltdelikte und ein erhöhter Risikofaktor für sexuell übertragbare Krankheiten. ${ }^{63}$

\section{Beischlaf unter Verwandten, $\S 173$ StGB}

Das StGB erstreckt sich auf die beiden elementaren Formen des Inzests, nämlich den Beischlaf zwischen Verwandten auf- und absteigender Linie ( $\mathbb{S} 173$ Abs. 1, Abs. 2 S. 1 StGB) mit geringerer Strafe für den Abkömmling, und sodann auf entsprechende sexuelle Handlungen unter leiblichen Geschwistern ( $\$ 173$ Abs. 2 S. 2 StGB), wobei das Gesetz eine Straffreistellung für diejenigen Abkömmlinge und Geschwister anordnet, die zur Tatzeit noch nicht volljährig waren ( $\mathbb{1 7 3}$ Abs. 3 StGB).

57 Lackner/Kübl, $\$ 171$ Rn. 2.

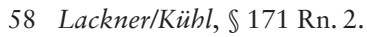

59 BGH NStZ 1992, 382.

60 Fischer, StGB, $\$ 171$ Rn. 4.

61 Lenckner/Bosch, in: Schönke/Schröder, $\$ 171$ Rn. 9.

62 ProstG v. 2 0.12. 2001 (BGBl. I S. 3983).

63 Renzikowski, GewArch 2008, 433. 
Das Gesetz stellt nach seinem eindeutigen Wortlaut auf die „Leiblichkeit“ (= genetischen Zusammenhang) der Beziehung, sowohl beim Eltern-Kind-Inzest als auch beim Geschwisterinzest ab. Eine strafbarkeitsausdehnende Auslegung der Norm, die nichtleibliche Kinder einbeziehen würde, wäre ein grober Verstoß gegen das Analogieverbot und damit verfassungswidrig. Dies bedeutet, dass $\$ 173 \mathrm{StGB}$ in modernen sozialen Patchwork- und Regenbogen-Familien nicht zur Anwendung gelangt, wenn es zum Beischlaf zwischen Patchworkelternteil und dem nicht leiblichen Kind oder zwischen Patchworkkindern kommt. Die Diskussion über die prinzipielle Legitimität des deutschen Inzesttatbestands, die vom BVerfG und dem EGMR bestätigt wurde, ${ }^{64}$ und seine tatbestandliche Ausgestaltung kann an dieser Stelle nicht geführt werden. ${ }^{65}$ Allerdings sind schon heute sexuelle Übergriffe in Patchwork- und Regenbogenfamilien strafrechtlich angemessen erfasst, weshalb insoweit keine nennenswerte strafrechtliche Schutzlücke besteht. Neben den Straftatbeständen, die bestimmte Sexualhandlungen per se pönalisieren - wie etwa der sexuelle Missbrauch von Kindern, von Jugendlichen oder die Vergewaltigung - und uneingeschränkt auch in „modernen“ Familien gelten, ist hier insbesondere an den Missbrauch von Schutzbefohlenen nach $\$ 174$ StGB zu denken.

\section{Sexueller Missbrauch von Schutzbefohlenen, §174 StGB}

Der Straftatbestand des sexuellen Missbrauchs von Schutzbefohlenen, $\mathbb{1 7 4}$ StGB, ist offen für die Einbeziehung von Kindern, die in einer Patchwork- oder Regenbogenfamilie leben. Das Gesetz knüpft für die Strafbarkeit von sexuellen Handlungen, die an oder vor dem Kind vorgenommen werden, maßgeblich an einen Täterstatus an, der darin besteht, dass das Kind zur Erziehung, Ausbildung oder Betreuung anvertraut wurde. Dazu gehört das Verhältnis des Pflegekinds zu seinen Pflegeeltern, aber auch dasjenige des Stiefkinds zum Stiefelternteil. ${ }^{66}$ Kinder in Patchworkfamilien werden dem Patchworkelternteil vielfach „ganzheitlich“, d. h. umfassend körperlich, geistig und sittlich zur Erziehung in der Lebensführung anvertraut sein; ${ }^{67}$ somit sind bei sexuellem Mißbrauchshandlungen in diesem Beziehungsgefüge folgerichtig auch die Straftatbestände der $\mathbb{S} 174$ Abs. 1 Nr. 1 bzw. Nr. 2 StGB mit ihren erhöhten Strafrahmen einschlägig. Insoweit wird die sexuelle Selbstbestimmung von Schutzbefohlenen auch in solchen gewandelten Familienformen geschützt.

\section{Förderung der sexuellen Handlungen Minderjähriger, §180 StGB}

Eltern, die sexuellen Handlungen ihrer unter 16 jährigen Kinder Vorschub leisten, indem sie eine entsprechende Gelegenheit verschaffen oder gewähren, machen sich

64 Vgl. BVerfGE 120, 224; EGMR NJW 2013, 215.

65 Zur Diskussion vgl. etwa Kubiciel, ZiS 2012, 282; Schramm (Fn. 1), S. 420 ff. alle m. w. N.

66 Dölling/Laue, in: Aman/Wipplinger (Hrsg.), Sexueller Missbrauch, 2005, S 891, 903; Lackner/Kühl, \$174 Rn. 7.

67 Vgl. etwa BGH NStZ 1998, 21; Renzikowski, MK-StGB, $\$ 174$ Rn. 16 (sofern nicht bloßes Zusammenleben in Hausgemeinschaft). 
nicht nach $\mathbb{1 8 0}$ Abs. 1 StGB strafbar, sofern sie durch dieses Vorschubleisten nicht gem. $\$ 180$ Abs. 1 S. 2 StGB ihre Erziehungspflicht gröblich verletzen. Das Gesetz knüpft für diese strafrechtliche Privilegierung, die strafrechtsdogmatisch einen Tatbestandsausschließungsgrund darstellt, ${ }^{68}$ an ein Handeln der "Sorgeberechtigten“ an. Dieser Kreis bestimmt sich nach BGB, meint also die Eltern ( $\$ 1626$ Abs. 1 BGB) - auch bei nichtehelicher Elternschaft (unter den Voraussetzungen des $\$ 1626$ a Abs. 1 BGB) -, bei Scheidung denjenigen, dem das Sorgerecht übertragen wurde ( $\mathbb{S} 1671,1672$ Abs. 1 BGB), den Vormund $(\mathbb{S} 1793$ BGB) und den Pfleger $(\mathbb{S} 1630$, 1688 BGB). Der Stiefelternteil ist nur dann - wenngleich mit Einschränkungen sorgeberechtigt, wenn er den leiblichen Elternteil geheiratet hat, wie sich aus $\mathbb{} 1687$ b Abs. 1 BGB ergibt. ${ }^{69}$ Ein echter sorgerechtlicher Status für Stiefeltern, d.h. eine vollständige Teilhabe an der elterlichen Sorge fehlt aber bis heute. Bei einer nichtehelichen Patchworkfamilie sieht das Gesetz ein solches partielles Sorgerecht gar nicht vor.

Zwar lässt das Zivilrecht die gänzliche Übertragung des Sorgerechts auf Dritte wegen dessen höchstpersönlicher Natur nicht zu, ${ }^{70}$ erlaubt aber Übertragung der Ausübung von elterlichen Sorgerechten und - pflichten auf Dritte, ${ }^{71}$ wobei hier vielfach von der Erteilung einer sog. Sorgerechtsvollmacht ausgegangen wird, die im Innenverhältnis auf einem Auftrags- oder Geschäftsbesorgungsverhältnis beruht. ${ }^{72}$ Dann stellt sich die Frage, ob in diesen Fällen neben Dritten (z. B. Verwandte, Schulen, Internate, Pflegeeltern) auch nichtverheiratete Patchwork-Mütter oder -Väter Personensorgeberechtigte i. S. von $\$ 180$ Abs. 1 S. 2 StGB sein können. Der Gesetzgeber hat sich im Gesetzgebungsverfahren mit der Frage der Einbeziehung Dritter beschäftigt, das aber ursprünglich vorgesehene „erweiterte Erzieherprivileg“, d. h. die Erstreckung von Abs. 1 S. 2 auf Dritte, die mit Einwilligung des Sorgeberechtigten handeln, auf Vorschlag des Vermittlungsausschusses gestrichen. ${ }^{73}$ Daraus wird überwiegend etwa abgeleitet, dass sich Dritte selbst dann strafbar machen, wenn sie auf das Geheiß des Sorgeberechtigten handeln, etwa wenn die Großmutter in Abwesenheit der Eltern, aber auf deren Weisung, die Tochter mit ihrem Freund ins Haus lässt. ${ }^{74}$

Doch kann hier richtigerweise eine analoge Anwendung der Norm auf nicht verheiratete Partner einer Patchworkfamilie nicht pauschal abgelehnt werden. Eine solche tatbestandliche Ausdehnung wäre verfassungsrechtlich unproblematisch, da es sich

68 Fischer, StGB, $\mathbb{1} 180$ Rn. 10; Schramm (Fn. 1), S. 286.

69 Statt aller Salgo, in: Staudinger, BGB, $\$ 1687$ b Rn. 8.

70 Peschel-Gutzeit, in: Staudinger, BGB, $\$ 1626$ Rn. 24.

71 Peschel-Gutzeit, in: Staudinger, BGB, $\$ 1626$ Rn. 28; Hamdan in: jurisPK-BGB, 6. Aufl. 2012, $\$ 1626$ BGB Rn. 9.

72 Götz, in: Palandt, BGB, 73. Aufl. 2014, $\$ 1626$ Rn. 3.

73 Renzikowski, MK-StGB, $\$ 180$ Rn. 43.

74 Renzikowski, MK-StGB, $\$ 180$ Rn. 43. 
um eine Analogie zugunsten des Täters handelt, die außerdem sowohl unter den eingangs skizzierten Dimensionen des verfassungsrechtlichen Familienschutzes als auch des Grundrechts auf Gleichbehandlung geboten sein kann. Insoweit müssen hier dieselben Überlegungen angestellt werden wie beim Zeugnisverweigerungsrecht. Nimmt jemand als Stiefelternteil in einer familiären Gemeinschaft de facto die Stellung eines Sorgeberechtigten ein, fällt sein Handeln in den Schutzbereich des (sozialen) Familienbegriffs in Art. 6 Abs. 1 GG, ohne das es auf das förmliche Sorgerecht ankommt. Dann kann aber sein Vorgehen nicht anders bewertet werden als dasjenige des leiblichen Elternteils. Wenn also der Patchwork-Vater es aufgrund seiner sorgerechtsähnlichen Stellung zulässt, dass in seinem Haus die 14 jährige Tochter seiner Lebensgefährtin mit ihrem ersten Freund geschlechtlich verkehrt, macht er sich demnach nicht nach $\mathbb{1} 180$ StGB strafbar, sofern sich dieses Gewähren nicht als gröblich Verletzung seiner sorgerechtsähnlichen Pflichten darstellt. Das gleiche gilt für die Erzieherprivilegien im Bereich der Gewaltdarstellung ( $\$ 131$ Abs. 4 StGB) und der einfachen Pornographie ( $\$ 184 \mathrm{StGB})$.

\section{Aussetzung, $\$ 221$ Abs. 2 StGB}

Auch wenn das Bild von der bösen Stiefmütter heutzutage weder zeitgemäß noch wahr ist, ${ }^{75}$ weckt das Thema Aussetzung in Patchwork Familien zwangsläufig Erinnerungen an das Grimm'sche Märchen von Hänsel und Gretel. Dort setzen die Eltern in großer finanzieller Not ihre beiden Kinder im Wald aus und überlassen sie ihrem Schicksal. Seit der Fassung des Märchens von 1840 ist es nicht mehr die eigene Mutter, auf deren Drängen die Kinder im Wald verlassen werden, sondern die Stiefmutter. $^{76}$

Nach heutigem StGB würde sich auch eine Stiefmutter (oder ein Stiefvater) einer Aussetzung nach $\mathbb{2} 21$ Abs. 1 StGB schuldig machen können. Jedermann, der eine andere Person in eine hilflose Lage versetzt, begeht eine Aussetzung nach Nr. 1 des $\$ 221$ Abs. 1 StGB, wenn er sie dadurch in die Gefahr des Todes oder einer schweren Gesundheitsschädigung bringt. Wird hingegen eine hilflose Person im Stich gelassen, fällt dies erst dann unter den Aussetzungstatbestand - und zwar unter Nr. 2 des $\$ 221$ Abs. 1 StGB, wenn der Unterlassende das Opfer in Obhut hat oder ihm ge-

76 Vgl. Brüder Grimm, Hänsel und Grethel, in: Kinder- und Haus-Märchen, 4. Aufl. 1840, Band 1: „(...) Vor einem großen Walde wohnte ein armer Holzhacker, der hatte wenig zu beißen und zu brechen, und kaum das tägliche Brot für seine Frau und seine zwei Kinder, Hänsel und Grethel. (...) „Hör, Mann, morgen in aller Frühe nimm die beiden Kinder, gib jedem noch ein Stückchen Brot, und führe sie hinaus in den Wald, mitten inne, wo er am dicksten ist, da mach ihnen ein Feuer an, und dann geh weg, und laß sie dort allein: wir können sie nicht länger ernähren.“ „Nein, Frau,“ sagte der Mann, „wie soll ich übers Herz bringen, meine eigenen lieben Kinder den wilden Thieren im Wald zu überliefern, die würden sie bald zerrissen haben.“. „Wenn du das nicht thust,“ sprach die Frau, „so müssen wir alle miteinander Hungers sterben,“ und ließ ihm keine Ruhe, bis er einwilligte. Die zwei Kinder waren auch noch vor Hunger wach gewesen, und hatten mit angehört was die Stiefmutter zum Vater gesagt hatte $(\ldots)^{*}$. 
genüber einer Beistandspflicht besitzt. Damit wird maßgeblich an die Figur des Beschützergaranten angeknüpft. ${ }^{77} \mathrm{Da}$ dieser Begriff- wie noch gleich zu zeigen sein wird - offen ist für „faktische“ oder „soziale Eltern“, aber auch „faktische“ Kinder, die sich um ihre Stief- oder Regenbogeneltern kümmern, sind in Patchworkfamilien auch Aussetzungen nach Nr. 2 des $\$ 221$ Abs. 1 StGB denkbar.

\section{Die Familie im Allgemeinen Teil des StGB}

Auch in den - „vor die Klammer“ der konkreten strafrechtlichen Tatbestände gezogenen - allgemeinen Voraussetzungen der Strafbarkeit können sich wichtige Fragestellungen mit Blick auf die Behandlung komplexer Familienformen ergeben. Im letzten Abschnitt dieses Beitrags sollen daraus drei Komplexe herausgegriffen werden: Begonnen wird mit dem sog. Angehörigenbegriff, der im Regelfall dazu dient, die Strafbarkeit oder zumindest die Verfolgbarkeit strafbaren Verhaltens einzuschränken; als zweites wird das Gewaltverbot in der Familie gestreift; am Ende soll auf etwaige Garantenstellungen innerhalb von Patchwork- und Regenbogenfamilien eingegangen werden.

\section{Der Angehörigenbegriff}

\section{a) Systematik}

Im Allgemeinen Teil des StGB spielen eheliche und familiäre Strukturen vordergründig nur in zwei Bereichen eine Rolle: Nach $\$ 11$ Abs. 1 Nr. 1 a) StGB gehören die Eltern und die Ehegatten wie auch eingetragene Lebenspartner zu den Angehörigen im Sinne des Strafrechts, einschließlich der Geschwister der Ehegatten, und zwar auch dann noch, wenn die Ehe nicht mehr besteht oder die Verwandtschaft erloschen ist. Hierin zeigen sich die Privilegien der Ehe und der Familie (sowie konsequent der eingetragenen Lebenspartnerschaft) gegenüber nichtehelichen Lebensgemeinschaften, Freundschaften oder anderen Formen persönlicher Verbundenheit. Das Verhältnis des Stiefelternteils gegenüber dem Stiefkind ist dann in den Angehörigenbegriff einbezogen, wenn der leibliche Vater bzw. die leibliche Mutter die Stiefmutter bzw. den Stiefvater geheiratet hat: Dann sind wie bereits mehrfach betont, Stiefkind und Stiefvater bzw. Stiefmutter miteinander verschwägert ${ }^{78}$ i. S. d. $\$ 1590$ BGB; die Schwägerschaft gerader Linie genügt als solche für $\$ 11$ Abs. 1 Nr. 1 a) StGB. Das bedeutet aber im Umkehrschluss: Die Partner einer nichtehelichen Lebensgemeinschaft, einer Patchworkfamilie oder Regenbogenfamilie stehen weder untereinander noch in Relation zu den leiblichen Kindern ihres Partners in einem Angehörigenverhältnis.

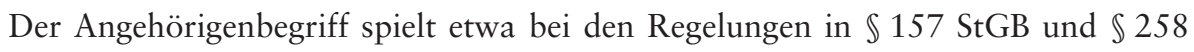
Abs. 6 BGB eine Rolle, die auf dem Gedanken der notstandsähnlichen Lage beru-

78 Statt aller Wellenhofer, in: MK-BGB, 6. Aufl. 2012, \$1590 Rn. 1. 
hen: ${ }^{79}$ So statuiert etwa der $\$ 258$ Abs. 6 StGB einen persönlichen Strafausschließungsgrund, der auf der Vorstellung beruht, dass der strafvereitelnde Beteiligte, der seinen Angehörigen der staatlichen Strafgewalt ausgesetzt sieht, sich in einer besonderen psychischen Zwangslage befindet ${ }^{80}$ und daher die Nachsicht der Rechtsordnung verdient, wenn ihm die familiäre Solidarität wichtiger ist als die staatliche Strafverfolgung.

Ebenso begegnet das Gesetz bei der Nichtanzeige geplanter Straftaten gem. $\$ 139$ Abs. 3 StGB dem Angehörigen in dem Fall mit Nachsicht, in dem er sich ernsthaft bemüht, den anderen von bestimmten Straftaten abzuhalten oder den Erfolg abzuwenden. ${ }^{81}$ Notstandsähnlich ist auch die Situation in $\mathbb{2} 213$ StGB: ${ }^{82}$ Hier sieht das Gesetz vor, dass ein minder schwerer Fall des Totschlags vorliegt, wenn der Totschläger sich aufgrund familiärer Empathie zur Tötung eines Provokateurs hat hinreißen lassen. Schließlich kommt auch der entschuldigende Notstand, $\mathbb{3} 35 \mathrm{StGB}$, bei Taten zugunsten Angehöriger in Betracht, wobei die Norm insoweit hinsichtlich der gefährdeten Personen offener als $\$ 157$ StGB und $\$ 258$ Abs. 6 StGB ausgestaltet ist, als nach $\mathbb{3} 35 \mathrm{StGB}$ auch Notstandstaten zugunsten „nahestehender Personen“ entschuldigt sein können. Untereinander nahestehend können sich - aufgrund persönlicher Verbundenheit ${ }^{83}$ - auch die Partner einer nichtehelichen Lebensgemeinschaft ${ }^{84}$ oder Stiefeltern und Stiefkinder sein.

\section{b) Analogiefähigkeit des Angehörigenbegriffs}

Allerdings wäre die Möglichkeit der Analogie beim formellen Angehörigenbegriff zugunsten des Täters in Betracht zu ziehen, wenn es sich um Partner einer Lebensgemeinschaft oder um eine familienähnliche Gemeinschaft handelt. Man denke nur an Fallkonstellationen, in denen der Täter aufgrund einer psychischen Konfliktsituation eine Straftat zugunsten seines Partners begeht, d. h. in den Fällen des $\$ 157$ StGB und $\$ 258$ Abs. 6 StGB. Wenn schon eine Notstandshandlung zugunsten einer nahestehenden Person in $\mathbb{3} 35$ StGB zur Entschuldigung führen kann, erscheint es mehr als diskutabel, bei den gleichen tatsächlichen Voraussetzungen zumindest den Aussagenotstand, $\mathbb{} 157$ StGB, sowie die notstandsähnliche Regelung des $\mathbb{} 258$ Abs. $6 \mathrm{StGB}$ auf nichteheliche Lebenspartner zu erstrecken. ${ }^{85}$ Nicht anders verhält es sich bei $\$ 213$ StGB. Bei den Strafantragsregelungen läge eine solche Analogie Rn. 73.

80 Cramer/Pascal, MK-StGB, $\mathbb{} 258$ Rn. 55.

81 Hohmann, MK-StGB, $\mathbb{1 3 9}$ Rn. 10: Unzumutbarkeit normgemäßen Verhaltens.

82 Eser, in: Schönke/Schröder, $\mathbb{S} 213 \mathrm{Rn} .1$.

83 Lackner/Kühl, \35 Rn. 4.

84 Perron, in: Schönke/Schröder, $\mathbb{S} 35 \mathrm{Rn} .11$.

85 So etwa Skwirblies, Nichteheliche Lebensgemeinschaft,1990, S. 138. 
ebenfalls nahe, ${ }^{86}$ wenn man bedenkt, dass $\$ 247$ StGB bei Entwendungen durch „in häuslicher Gemeinschaft Lebende“ einen Strafantrag vorsieht. Man wende dagegen nicht prozessuale Nachweisschwierigkeiten und eine damit verbundene Missbrauchsgefahr ${ }^{87}$ ein: denn diese praktischen Problem bestehen auch bei $\$ 35 \mathrm{StGB}$ und $\mathbb{2} 247$ StGB, die der Gesetzgeber offensichtlich in Kauf genommen hat.

Freilich liegen keine planwidrige Regelungslücke und damit an sich keine analogielegitimierende Situation bei denjenigen Vorschriften vor, in denen sich der Gesetzgeber bewusst beschränkt hat und die einen abschließenden Charakter besitzen. Da-

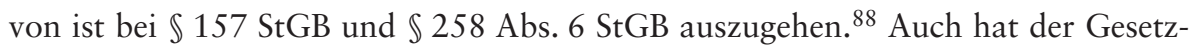
geber, als er den Angehörigenbegriff auf die eingetragene Lebenspartnerschaft ausdehnte, die nichtehelichen Lebensgemeinschaften nicht in den Angehörigenbegriff integriert, obwohl ihm im Jahre 2002 das Phänomen der nichtehelichen Lebensgemeinschaft bekannt war. Auch aufgrund des gesetzgeberischen Verzichts auf eine Stellungnahme zu den nichtehelichen Lebensgemeinschaften liegt die Vermutung nahe, dass insoweit alles beim Alten geblieben ist ${ }^{89}$ und somit eine analoge Anwendung des Angehörigenbegriffs auf nichteheliche Lebensgemeinschaften, Patchworkfamilien und Regenbogenfamilien, soweit es nicht das Verhältnis zwischen dem leiblichem Elternteil und seinen Kind betrifft, derzeit nicht in Betracht kommt. ${ }^{90}$

Gleichwohl stellt sich die Frage, ob man es dabei belassen kann und mit solchen rechtsmethodischen Überlegungen die Entscheidung des Gesetzgebers als verfassungsrechtlich vertretbare Entscheidung hinzunehmen muss - oder ob nicht doch mit Blick auf den verfassungsrechtlichen Schutz der Familie und das Gleichheitsgebot eine analoge Anwendung der genannten Bestimmungen auf Patchwork- und Regenbogenfamilien ernsthaft in Betracht gezogen werden sollte.

Hinsichtlich des Strafantragsrechts erscheint mir dies freilich problematisch: Denn im Vergleich zu entsprechenden Problematik bei den Zeugnis- und Aussageverweigerungsrechten muss zunächst hervorgehoben werden, dass das Gesetz ein Strafantragsrecht des Angehörigen nur bei bestimmten Straftatbeständen vorsieht, nicht jedoch pauschal. Mithin können schon heute Angehörige die Strafverfolgung nur bei einem relativ kleinen Kreis von Delikten verhindern. Dagegen ist das Zeugnis- und Aussageverweigerungsrecht nicht auf bestimme Delikte beschränkt, sondern gilt für alle Zeugenaussagen, mag der Vorwurf bloß Hausfriedensbruch ( $\$ 123 \mathrm{StGB}$ ) oder sogar Völkermord ( $\$ 6$ VStGB) lauten. Darin kommt zugleich die besondere, fundamentale Bedeutung und „Dramatik“ des Zeugnisverweigerungsrechts zum Aus- 
druck: Bei einer Zeugenaussage liefert der Zeuge den beschuldigten Angehörigen möglicherweise „an das Messer“ der Strafjustiz; bei einem Antragsdelikt hingegen hat der Angehörige ein Veto-Recht hinsichtlich der Strafverfolgung, wenn er selbst von der Tat als Verletzter betroffen ist. Insofern handelt es sich hier um unterschiedliche Fallkonstellationen und Interessenlagen, die nicht auf einen gemeinsamen Nenner gebracht werden können und müssen. Im übrigen kann auch bei einem Antragsdelikt der verletzte Angehörige die Strafverfolgung faktisch verhindern, in dem er entweder den Sachverhalt nicht anzeigt oder, wenn die Verfolgungsorgane anderweitig von der Straftat erfahren, sich dann bei der Zeugenvernehmung auf sein Zeugnisverweigerungsrecht beruft.

Hinsichtlich der Sachverhalte, in denen das Angehörigenprivileg im Zusammenhang mit einer Schuldmilderung steht, wie etwa bei $\mathbb{S} \$ 139$ Abs. 3, 157, 213, 258 Abs. 6 $\mathrm{StGB}$, ist jedoch - entgegen der herrschenden Meinung ${ }^{91}$ - eine analoge Anwendung des Angehörigenbegriffs auf nahestehende Personen zu bejahen: Dafür spricht nicht nur die insgesamt zu beobachtenden Tendenz zur Gleichstellung naher nichtverwandtschaftlicher Beziehungen mit verwandtschaftlichen, ${ }^{92}$ sondern - gewichtiger der in solchen Beziehungen gleichermaßen ausgelöste seelische Zwang, der ganz demjenigen eines „Blutsverwandten“ oder Ehe- bzw. Lebenspartner entsprechen kann. ${ }^{93}$ Die Erwägungen sind letztlich dieselben wie oben (II. 1., 2) beim Zeugnisund Auskunftsverweigerungsrecht.

\section{Gewaltverbot in der Familie; stellvertretende Einwilligung \\ a) Recht auf gewaltfreie Erziehung}

Nach $\mathbb{1 6 3 1}$ Abs. 2 S. 1 BGB haben Kinder ein Recht auf gewaltfreie Erziehung. Dieses Gewaltverbot in der Familie richtet sich, wie die Überschrift des $\$ 1631$ BGB zeigt („Umfang der Personensorge“), an die Personensorgeberechtigten, deren Kreis bereits oben (III. 6) umrissen wurde. Sollten Stiefeltern, Lebenspartner usw. nicht förmlich sorgeberechtigt oder ihnen Erziehungsbefugnisse auch nicht von den Eltern übertragen worden sein, ${ }^{94}$ so kann dies freilich nicht heißen, dass sie an das Gewaltverbot nicht gebunden sind. Vielmehr handelt es sich hier um eine prinzipielle Aussage zur Art und Weise, mit welchen Mitteln Kinder heute nicht mehr erzogen werden dürfe, weshalb das Gewaltverbot erst recht an diejenigen richtet, die faktisch Träger der Personensorge sind. Insoweit ist $\$ 1631$ BGB analog auf „Eltern im sozialen Sinne" anwendbar.

91 Vgl. etwa zu $\mathbb{} 157$ StGB BayObLG NJW 1986, 202; Lenckner/Bosch, in: Schönke/Schröder, $\mathbb{} 157$ Rn. 6; zu $\ 258$ Abs. 6 StGB BGH NJW 1984, 135, 136; Kindhäuser, LPK, $\$ 258$ Rn. 23;

92 Neumann, NK-StGB, $\$ 213$ Rn. 11.

93 Ebenso zu $\$ 213$ StGB Fischer, StGB, 61. Aufl. 2014, $\$ 11$ Rn. 10; zu $₫ 157$ StGB Vormbaum, NK-

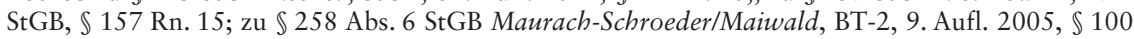
Rn 24

94 Huber, MK-StGB, $\$ 1631$ Rn. 29. 
Man sollte die rechtliche Relevanz dieser Analogie für das Strafrecht freilich nicht übertonen. Wenngleich hier nicht der Ort ist, im Detail auf den Umfang des (stief-)elterlichen Erziehungsrechts einzugehen, bleibt zu beachten: Das Züchtigungsrecht alten Zuschnitts, das den Eltern bei entsprechenden Anlass körperliche Eingriffe bis hin zu Schlägen auf das Hinterteil oder kräftigen Ohrfeigen gestattet hatte, existiert - nach herrschender Meinung - ohnehin nicht mehr, weshalb auch keine Übertragung des Züchtigungsrechts möglich ist: ein Nullum ist nicht übertragbar. ${ }^{95}$ Allerdings bejaht ein Teil des Schrifttums, mit unterschiedlicher Begründung und Reichweite, weiterhin ein Züchtigungsrecht. ${ }^{96}$

\section{b) Stellvertretende Einwilligung}

Inwieweit Eltern stellvertretend eine Einwilligung (etwa in einen operativen Eingriff) erteilen (bzw. verweigern) können, sofern das minderjährigen Kind nicht selbst über den Eingriff entscheiden kann und insofern nicht die alleinige Zuständigkeit besitzt, hängt prinzipiell davon $a b$, ob der Minderjährige nach seinem Entwicklungsstand die Bedeutung und die Tragweite des Eingriffs zu beurteilen vermag, mithin eine ausreichende Einsichtsfähigkeit besitzt. Ist dies nicht der Fall, wird allein der förmlich Sorgeberechtigte über die Einwilligung befinden können. Der Umfang und die Grenzen des verfassungsrechtlichen garantierten Sorgerechts sind auch hier von Bedeutung, wie sie etwa zuletzt leidenschaftlich im Zusammenhang der Zirkumzision von Knaben diskutiert wurden ${ }^{97}$ und auch beim neuen Straftatbestand der weiblichen Genitalverstümmelung ( $\$ 226$ a StGB) thematisiert werden müssen. ${ }^{98}$ Die Entscheidungszuständigkeit bei Einwilligungen wird vom förmlichen Sorgeberechtigten auch dem nichtleiblichen Patchworkelternteil übertragen werden können, allerdings nur, soweit dies nach den familienrechtlichen Vorgaben möglich ist. ${ }^{99}$

\section{Garantenstellungen, $§ 13 \mathrm{StGB}$}

Im Bereich der unechten Unterlassungsdelikte ist ebenfalls eine weitgehende Einbeziehung der sozialen Eltern möglich und angezeigt. Hierzu sei zunächst an die Grundaussage des $\mathbb{1} 13$ StGB erinnert: Ein (explizit oder inzident) allein auf aktives Tun zugeschnittener Straftatbestand kann dann ausnahmsweise auf einen Unterlassens-

95 Vgl. Salgo, in: Staudinger, $\$ 1631$ BGB Rn. 90.

96 Zur Diskussion über das Ob und Wie des elterlichen Züchtigungsrechts vgl. etwa Kübl, StrafR AT, 7. Aufl. $\$ 18$ Rn. 74 ff.; Schramm (Fn. 1), S. 207; für Beibehaltung des Züchtigungsrechts in engen Grenzen unter Berufung auf die Verfassungswidrigkeit des $\$ 1631$ BGB im Lichte des elterlichen Erziehungsrechts (Art. 6 Abs. 2 GG) etwa Murmann, Grundkurs Strafrecht, 2. Aufl. 2013, $\mathbb{2 5}$ Rn. 151 ff. m. w. N.

97 Vgl. etwa Fateh-Moghadam, RW 2010, 115; Germann, MedR 2013, 412; Hörnle/Huster JZ 2013, 328; Scheinfeld, HRRS 2013, 268.

98 Vgl. etwa Schramm, Festschrift für Kristian Kühl, 2014, S. 632.

99 Zur Übertragung von Sorgerechtsbefugnissen vgl. Dethloff, Familienrecht, 30. Aufl. 2012, \$13 Rn., 19; Peschel-Gutzeit, in: Staudinger, BGB, $\$ 1626$ Rn. 24 (in der Substanz unübertragbares Sorgerecht), Schwab, Familienrecht, 21. Aufl. 2013, Rn. 698. 
täter angewendet werden, wenn er nach $\mathbb{1 3} \mathrm{StGB}$ rechtlich dazu verpflichtet ist, einen tatbestandsmäßigen Erfolg abzuwenden.

\section{a) Garantenstellung der Eltern gegenüber dem Kind}

Blickt man zunächst auf das Eltern-Kind-Verhältnis, ist festzuhalten: Leibliche Eltern haben gegenüber ihren minderjährigen Kindern im Regelfall eine Garantenstellung: Sie sind zum einen dazu verpflichtet, ihre Kinder zu beschützen und Gefahren für die Rechtsgüter ihrer Kinder abzuwenden (Beschützergaranten), ${ }^{100}$ aber auch dafür zu sorgen, dass sie ihre Kinder überwachen und von ihren Kindern keine Gefahren für die Rechtsgüter anderer ausgehen (Überwachungsgarantenstellung). ${ }^{101}$ Diese strafrechtliche Handlungspflicht wird meist - in einer institutionellen Betrachtungsweise - aus der familienrechtlichen Verpflichtung zur elterlichen Sorge aus $\$ 1626$ Abs. 1 S. 1 BGB und ihrer Konkretisierung in $\mathbb{1 6 3 1}$ Abs. 1 BGB abgeleitet. Ihr verfassungsrechtliches Fundament bildet das „Pflichtenrecht“ des Art. 6 Abs. 2 GG. Sind die Kinder dagegen erwachsen, wird im Regelfall keine Überwachungsgarantenstellung mehr bestehen, und auch eine Beschützergarantenstellung kommt dann nur noch in Betracht, wenn Eltern und Kinder eine häusliche Gemeinschaft in Gestalt einer familiären Lebensgemeinschaft bilden. ${ }^{102}$ Für nichteheliche Lebensgemeinschaften mit gemeinsamen leiblichen Kindern ergibt sich das Sorgerecht nach der Maßgabe des $\$ 1626$ a BGB.

Schwieriger zu beurteilen ist dagegen die familienrechtliche Ausgangssituation bei Patchwork- und Regenbogenfamilien ohne gemeinsame leibliche Kinder und ohne förmliche eheliche bzw. lebenspartnerschaftliche Bindung der Partner. Zwischen Stiefeltern und Stiefkindern in nichtehelichen Lebensgemeinschaften oder nicht eingetragenen Lebenspartnerschaften besteht, wie sich im Umkehrschluss aus $\$ 1687 \mathrm{~b}$ BGB ergibt, kein formelles sorgerechtliches Band, ${ }^{103}$ so dass $\mathbb{1 6 2 6}$ und $\mathbb{1 6 2 6 \mathrm { a }}$ BGB als Begründung für eine Garantenstellung der Patchworkeltern nicht in Betracht kommen. Soziale Elternschaft reicht für das Sorgerecht gerade nicht aus. ${ }^{104}$ Mithin kann es hier zu einem Auseinanderfallen von Lebenswirklichkeit und sorgerechtlicher Verantwortungszuweisung kommen.

Handelt es sich um eine Stieffamilie, innerhalb derer die ein Kind in die eheliche Familiengemeinschaft aufgenommen wird, für das nur einer der Ehepartner das Sorgerecht hat, kann aber zivilrechtlich der Sorgeberechtigte einen Teil seiner Sorgerechte und Pflichten einem Dritten und damit auch dem Stiefelternteil übertragen. Es

100 Kühl, StrafR AT, 7. Aufl., $\$ 18$ Rn. 48

101 Kühl, StrafR AT, $\$ 18$ Rn. 116. Zur Funktionenlehre von Armin Kaufmann, der diese Kategorien „Beschützer- und Überwachungsgarantenstellung“ entwickelt hat, vgl. Roxin, StrafR AT-2, 2003, $\$ 32 \mathrm{Rn} .6$

102 Brückner, Das Angehörigenverhältnis der Eltern im Straf- und Strafverfahrensrecht, 2000, S. 66.

103 Salgo, in: Staudinger, BGB, $\$ 1687$ b Rn. 8.

104 Peschel-Gutzeit, in: Staudinger, BGB, $\$ 1626$ Rn. 32. 
kommt dann nach herrschender Meinung ${ }^{105}$ zu einer Aufsichtspflicht aufgrund vertraglicher Übernahme. In der Kommentarliteratur wird hierzu betont, dass die Personensorgerechtsbereiche des $\mathbb{1 6 3 1}$ Abs 1 wie Pflege, Erziehung und Aufsicht häufig von den Eltern zur Ausübung auf Dritte übertragen werden, z. B. auf die "Kinderfrau“, bei Tagesbetreuung wie Krippe, Kindergarten, Hort, Tagespflege. Für die Übertragung auf den Partner kann nichts anderes gelten.

Darüber hinaus schreibt das Zivilrecht vor, dass Stiefeltern familienrechtliche Verantwortung für die Kinder übernehmen, soweit sich eine entsprechende Zuständigkeit aus der gelebten Rollenverteilung in der Beziehung ergibt. Daraus folgt zunächst ein Umgangsrecht nach $\mathbb{\$} 1685$ Abs. 1BGB, sofern eine vom Gesetzgeber geforderte sozial-familiäre Beziehung besteht. ${ }^{106}$ Es kann sogar gem. $\$ 1682$ BGB vom Familiengericht angeordnet werden, dass in den Fällen, in denen infolge des Todes des leiblichen Elternteils das elterliche Sorgerecht einschließlich des Aufenthaltsbestimmungsrechts auf den anderen leiblichen Elternteil übergeht, das Kind beim Stiefelternteil verbleibt, wenn es längere Zeit mit dem leiblichen und dem Stiefelternteil in einem Haushalt gelebt hat und das Kindeswohl durch die Wegnahme gefährdet würde.

Ferner haben diese übernommenen Aufsichtspflichten Konsequenzen für die deliktische Haftung gem. $\$ 832$ Abs. 2 BGB, die eben auch ein Patchworkelternteil treffen kann, sofern er vertraglich eines solche Aufsichtspflicht übernommen hat. Letzteres ist zu bejahen, wenn ein Stiefkind dauerhaft in die häusliche Gemeinschaft aufgenommen wird. ${ }^{107}$ Mit der vertraglichen Begründung einer Aufsichts- und Schutzpflicht lässt sich zwanglos eine Brücke zur rechtlichen Einstandspflicht nach $\mathbb{} 13$ StGB schlagen, als damit eine Beschützer- und Überwachungsgarantenstellung kraft Übernahme (Vertrag) begründet wird. Diese Übernahme erfolgt regelmäßig stillschweigend und bedarf keiner weiteren ausdrücklichen, vertraglichen und entgeltlichen Regelungen. ${ }^{108}$

Nach herrschender Meinung im strafrechtlichen Schrifttum kommt es in diesen Bereich ohnehin nicht entscheidend auf eine entsprechende familien- oder verfassungsrechtliche Begründung der Garantenstellung an, da diese „nicht den tragenden Grund für die Entstehung von Obhutspflichten " 109 bilde. Vielmehr sei bei solchen Gemeinschaftsverhältnissen maßgeblich deren „Angelegtsein auf gegenseitigen Beistand in der Not“. ${ }^{110}$ Hierfür sei keine ausdrückliche Zusicherung wechselseitigen

105 OLG Düsseldorf NJW-RR 1992, 857; Wagner, MK-BGB, $\$ 832$ Rn. 11.

106 Muscheler, FamRZ 2004, 913.

107 OLG Jena OLGR Jena 2002, 381; Beling, in: Staudinger, BGB, $\$ 832$ Rn. 41; Wagner, in: MK-BGB, \$832 Rn. 11 .

108 Vgl. OLG Düsseldorf (Fn. 105).

109 Kühl, AT, $\$ 18$ Rn. 61.

110 Kühl, AT, $\$ 18$ Rn. 62. 
Beistands erforderlich; vielmehr genügen die konkludent formulierte Erwartungshaltung und ein entsprechendes gegenseitiges Vertrauen. ${ }^{111}$

Man mag gegen eine solche Betrachtungsweise einwenden, dass damit letztlich nur - möglicherweise diffuses - ethisch gefordertes Verhalten zu einer umdeklarierten Rechtspflicht werde. Gleichwohl greifen diese Bedenken hier nicht durch. Denn es gibt durchaus Schnittmengen zwischen der Ethik (der guten Sitten, der Moral) und rechtlichen Geboten bzw. Verboten. Abgesehen davon konstruiert das Zivilrecht zwar nicht auf der Grundlage eines Vertrags oder einer ausdrücklichen speziellen gesetzlichen Vorgabe, aber doch - im Rahmen der allgemeinen deliktischen Haftung nach $\mathbb{8} 823$ Abs. 1 BGB (und nicht lediglich akzessorisch zu Straftatbeständen als Schutzgesetze i. S. d. $\$ 823$ Abs. 2 BGB) eine originäre zivilrechtliche Handlungspflicht etwa bei enger Lebensgemeinschaft. ${ }^{112}$

Richtigerweise handelt es sich somit bei der Obhutsgarantenstellung auch in der nichtehelichen Lebensgemeinschaft, der Patchworkfamilie oder Regenbogenfamilie um eine allgemeine (zivil-)rechtliche Pflicht zur Solidarität, die auch strafrechtlich flankiert und sanktioniert wird. Zudem wird man, wie bereits angedeutet, vielfach sogar davon ausgehen können, dass der nicht sorgeberechtigte Partner kraft Vertrags personensorgerechtsähnliche Aufgaben in Bezug auf das minderjährige Kind übernommen hat.

\section{b) Verhältnis des Kindes gegenüber Eltern}

Schwieriger zu beantworten ist die Frage, inwieweit Stief-/Patchwork-/Regenbogenkinder zum rechtlichen Schutz ihrer Stiefeltern (und zum Schutz gefährdeter Dritter vor ihren Stiefeltern) verpflichtet sind. Hier wird man wiederum zwischen der Überwachungs- und Beschützergarantenposition unterscheiden müssen. Schon im Verhältnis der leiblichen Kinder zu ihren Eltern wird man mit der Annahme einer Überwachungsgarantenstellung sehr vorsichtig sein und ihr letztlich ablehnend gegenüber stehen müssen: Soweit Eltern eigenverantwortlich andere gefährden oder verletzen, tragen Kinder keine Verantwortung dafür, gleichsam eine familiäre Polizisten-Rolle einzunehmen und die Eltern von ihrem „kriminellen Treiben“ abzuhalten. ${ }^{113}$ Erst recht begründet die Schwägerschaft nach $\ 1590$ BGB als solche keine Garantenstellung. ${ }^{114}$

Eine Beschützergarantenstellung wird man den Stiefkindern jedoch dann auferlegen müssen, wenn die Kinder die Betreuung ihrer gebrechlichen oder kranken Stiefeltern übernommen haben oder mit ihnen in einer häuslichen Gemeinschaft leben. Insoweit

111 Vgl. Kühl, AT, $\$ 18$ Rn. 62 m. w. N.

112 Vgl. Hager, in: Staudinger, BGB, $\mathbb{} 823$ Rn. 6.

113 Schramm (Fn. 1) S. 261.

114 OLG Düsseldorf NJW-RR 2000, 1623. 
kann nichts anderes gelten als im Verhältnis von leiblichem Kind zu den Eltern. ${ }^{115}$ Zwar kann in diesem Fall die Garantenstellung nicht auch auf $\$ 1618$ a BGB gestützt werden, da sich die Pflicht zum Beistand auf das Verhältnis von leiblichen Eltern und Kindern bezieht. Auch kann nicht die „natürliche“, d. h. genetische Abstammung als solche die Grundlage für eine Beschützergarantenstellung bilden. Wenn aber die Verbundenheit derjenigen einer „klassischen“ Familie entspricht, sich insbesondere mit der - über etwa das bloße Zusammenwohnen hinausgehende - engen Verbundenheit der Familienangehörigen ausdrücklich oder konkludent die Erwartung verbindet, dass man sich in der Not solidarisch gegenüber seinen Stiefeltern verhält, handelt es sich hierbei nicht nur um eine ethischen Verpflichtung, sondern zugleich um eine rechtliche Pflicht (nicht nur im Sinne des $\mathbb{8} 823$ Abs. 1 BGB, sondern auch) i. S. d. $\$ 13$ StGB. In solchen Fällen wird man, zumindest bei erwachsenen Stiefkindern, zuweilen sogar von einer Beschützergarantenstellung kraft konkludent geschlossenen Vertrags ausgehen können.

\section{c) Verhältnis der Geschwister untereinander}

Noch schwieriger zu beurteilen ist die Frage, ob die Geschwister einer Patchworkfamilie untereinander eine Beschützer- oder Überwachungsgarantenstellung einnehmen. Muss der Stiefbruder verhindern, dass seine Stiefschwester Diebstähle begeht? Macht sich die Stiefschwester nicht bloß wegen unterlassener Hilfeleistung ( $\$ 323 \mathrm{c}$ StGB), sondern sogar wegen fahrlässiger Tötung durch Unterlassen $(\mathbb{S} 222,13$ StGB) strafbar, wenn sie nicht den Notarzt ruft, obwohl ihr Stiefbruder komatös mit einer Alkohol- und Marihuanavergiftung im Bett liegt und daran stirbt, weil sie keinen Arzt geholt hat? Blickt man hier wieder zunächst auf $\$ 1618$ a BGB, so ist festzuhalten, dass nach ganz h. M. die darin zum Ausdruck gebrachte Solidaritätspflicht nicht auf Stiefkinder anwendbar ist, und zwar selbst dann nicht, wenn der leibliche Elternteil den Stiefelternteil geheiratet hat, da die Schwägerschaft ebenso wenig wie rein psychologische Eltern-Kind-Beziehungen hierfür genügen. ${ }^{116}$ Etwas anderes gilt aber für Pflegekinder, da deren tatsächliche Familienbeziehung in $\$ 1688$ BGB rechtlich geregelt ist, auch wenn hier kein „vollwertiges“ Eltern-Kind-Verhältnis bestünde. ${ }^{117}$ Zumindest kann eine Überwachungs- und Beschützergarantenstellung des volljährigen Stiefgeschwisterteils kraft Delegation angenommen werden, wenn ihm die Wahrnehmung der sorgerechtlichen Aufgaben von den Eltern (volles oder kleines Sorgerecht) explizit oder konkludent und damit rechtsgeschäftlich übertragen wurden. Dann begründet jedoch erst diese Vereinbarung, nicht jedoch schon das bloße Zusammenleben in einer Patchworkfamilie als solches die Garantenstellung.

115 Schramm (Fn. 1) S. 261.

116 Coester, in: Staudinger, BGB, $\mathbb{} 1618$ a Rn 25.

117 Coester, in: Staudinger, BGB, $\mathbb{S} 1618$ a Rn 24. 


\section{Fazit und Ausblick}

$\mathrm{Ob}$ das Strafrecht der veränderten Wirklichkeit heutiger Formen der Familienzusammensetzung und dem Wandel des Familienbegriffs de lege lata gerecht werden kann, hängt zunächst von der tatbestandlichen Ausgestaltung der Strafnorm bzw. der Regelung im Allgemeinen Teil des StGB zusammen. Offen für die Einbeziehung von Patchwork- oder Regenbogenfamilien sind etwa die Regelungen zur Garantenstellung ( $\$ 13 \mathrm{StGB}$ ), zum Strafantragserfordernis bei Straftaten im Haus und der Familie $(\mathbb{S} 247 \mathrm{StGB})$ oder solche Bestimmungen, in denen eine faktische Nahebeziehung etwa in Gestalt der nahestehenden Person in das Gesetz integriert wurde ( $\int 35$ StGB). Die für viele Privilegierungen und Qualifikationen maßgebliche Figur des Angehörigen erstreckt sich, wie sich aus dem Wortlaut des $\mathbb{1 1}$ Abs. 1 Nr. 1 StGB ergibt, zwar auch auf das Verhältnis zwischen nichtehelich geborenem Kind und seinen leiblichen Eltern sowie auf das Verhältnis Stiefeltern-Stiefkind, sofern aufgrund einer Heirat eine Schwägerschaft entstanden ist, aber nicht auf das Verhältnis von Stiefeltern zu Stiefkindern, sofern der Stiefelternteil nicht den leiblichen Elternteil heiratet. Zwar braucht man sich dann prinzipiell nicht mit einem gegenwärtigen Gesetzeszustand abzufinden, sondern könnte über eine an der verfassungsrechtlichen Schutzverpflichtung gegenüber der (sozialen) Ehe ausgerichtete verfassungskonforme Interpretation diesen im Wege einer Analogie in bonam partem korrigieren, wenn es sich um eine planwidrige Gesetzeslücke handelt; aber diese „Lücke“ besteht nach den kriminalpolitischen Vorstellungen des Gesetzgeber im Allgemeinen Teil des StGB offensichtlich nicht und wäre daher an sich nicht planwidrig. Solange aber der Gesetzgeber de lege ferenda den status quo des $\mathbb{} 11$ Abs. $1 \mathrm{Nr} .1$ StGB nicht ändert, ist zumindest in denjenigen Regelungsbereichen, in denen vergleichbare seelische Konflikte auftreten können wie im Verhältnis von leiblichem Elternteil und Kind, eine analoge Anwendung des Angehörigenbegriffs zu bejahen.

Die Pluralität und Diversität heutiger Familienformen eröffnet nicht nur neue Perspektiven persönlicher und familiärer Lebensentfaltung und -gestaltung, sie stellen auch die Gesellschaft und das Recht vor Herausforderungen. Es ist zwar nicht die Aufgabe des Strafrechts, in diesen Bereichen eine rechtliche Vorreiterstellung einzunehmen. Wegen der vielfach explizit oder inzident zivil- und familienrechtsakzessorischen Natur strafrechtlicher Regelungen ist ohnehin primär das Zivilrecht dazu aufgerufen, die Verbote und Gebote, die für solche Familienformen gelten sollen, zu definieren. Nur soweit in der Primärrechtsordnung des BGB, freilich unter korrigierender Einbeziehung des höherrangigen Verfassungsrechts, die Rechte und Pflichten in Stief-, Patchwork- und Regenbogenfamilien definiert sind (etwa in Gestalt von Unterhaltsverpflichtungen oder Sorgerechten), kann das Strafrecht diese außerstrafrechtlichen Regelungsmechanismen mit einem begleitenden Schutz vor dem und durch das Strafrecht verbinden. 
Das Strafrecht kann und darf sich im Übrigen der familienkulturellen Entwicklung nicht verschließen. Eine Diskriminierung von nichtehelich geprägten Familienformen, von Patchworkfamilien heterosexueller Partner und von Regenbogenfamilien schwuler und lesbischer Partner verbietet sich. Vielmehr muss die Rechtsordnung die modernen Familienformen genauso vor dem Strafrecht und durch das Strafrecht schützen wie die klassische Familie, sofern erstens der soziale Familienbegriff des Art. 6 Abs. 1 GG zu verfassungskonformen Anpassungen der strafrechtlichen Sanktionsordnung Anlass gibt, zweitens kein sachlicher Grund für eine am Maßstab des Art. 3 Abs. 1 GG gemessene Ungleichbehandlung von klassischen und modernen Familienformen vorliegt und drittens darüber hinaus bereits die übrige Rechtsordnung, namentlich das Zivil- und Familienrecht, bei vergleichbaren Interessenkonflikten schon heute (zumindest ansatzweise) Patchwork- und Regenbogenfamilien ihre Anerkennung nicht versagt. Daher sprechen beispielsweise beim Zeugnis- und Aussageverweigerungsrecht die besseren Gründe dafür, diese auf das Verhältnis von (nichtverheiratetem) Stiefelternteil und Stiefkindern sowie von Patchworkfamilien und Regenbogenfamilien zu erstrecken, dies freilich nicht pauschal, sondern dann, wenn deren gelebte Beziehungswirklichkeit derjenigen einer intakten Kern-Familie entspricht. 